\title{
Many-body theory of positron-atom interactions
}

\author{
G. F. Gribakin $*$ and J. Ludlow \\ Department of Applied Mathematics and Theoretical Physics, \\ Queen's University, Belfast BT7 1NN, Northern Ireland, UK
}

(Dated: August 24, 2018)

\begin{abstract}
A many-body theory approach is developed for the problem of positron-atom scattering and annihilation. Strong electron-positron correlations are included non-perturbatively through the calculation of the electron-positron vertex function. It corresponds to the sum of an infinite series of ladder diagrams, and describes the physical effect of virtual positronium formation. The vertex function is used to calculate the positron-atom correlation potential and nonlocal corrections to the electron-positron annihilation vertex. Numerically, we make use of B-spline basis sets, which ensures rapid convergence of the sums over intermediate states. We have also devised an extrapolation procedure that allows one to achieve convergence with respect to the number of intermediate-state orbital angular momenta included in the calculations. As a test, the present formalism is applied to positron scattering and annihilation on hydrogen, where it is exact. Our results agree with those of accurate variational calculations. We also examine in detail the properties of the large correlation corrections to the annihilation vertex.
\end{abstract}

PACS numbers: 34.85.+x, 31.15.Lc, 34.10.+x, 78.70.Bj

\section{INTRODUCTION}

The interaction of a low-energy positron with a many-electron atom is characterised by strong correlation effects. Apart from the dynamic polarisation of the electron cloud by the field of the positron, the positron can also form positronium (Ps), by picking up one of the atomic electrons. When the positron energy is below the Ps-formation threshold, $\varepsilon_{\mathrm{Ps}}=I+E_{1 s}(\mathrm{Ps})=I-6.8 \mathrm{eV}$, where $I$ is the atomic ionisation potential, positronium formation is a virtual process. Nevertheless, its role in the positron-atom interaction is very important [1, 2, 3, 4]. The main aim of this work is to develop a many-body theory approach which accounts accurately for both correlation effects, and to test it for positron scattering and annihilation on hydrogen.

The study of positron interaction with matter is a topic of fundamental interest [5]. Positrons have also found many useful applications. They are a very sensitive probe of the presence of defects in materials $\underline{6}$. The recent development of a scanning positron microscope 7] may lead to an increased use of positrons for quality control of materials, particularly in the semiconductor industry. In medicine, positron emission tomography, or PET, has become a standard means of medical imaging (see, e.g., [ 8$]$ ). A proper understanding of how positrons interact with matter at the fundamental level of atoms and molecules, is therefore essential.

The interaction of low-energy positrons with atoms has presented a challenge to the theorist for many decades. The study of positron scattering from atoms was initially seen as a useful complement to work on electron scattering, particularly in helping to understand the role of the exchange interaction. However, although the exchange interaction is absent, it was quickly realised that the positron-atom problem is more complex than the electron case. The attractive induced polarisation potential tends to cancel and even overcome the static repulsion of the positron by the atom at low energies. The positron may also temporarily capture one of the atomic electrons in a process known as virtual positronium formation. The need to account for these effects requires an elaborate and accurate theoretical description.

For small systems, such as hydrogen and helium, accurate results were obtained through the use of variational methods 9, 10, 11, 12, 13, 14]. Positron scattering from alkali atoms which have a single valence electron, has been calculated extensively using a coupled-channel method with pseudostates [15]. More recently, positron and Ps interaction with atoms with few active (valence) electrons has been studied using the stochastic variational method (SVM) and configuration-interaction-type approaches [16, 17, 18, 19, 20]. However, it is difficult to extend these methods to larger atomic systems with many valence electrons, e.g., the noble gases.

An attractive alternative to few-body methods is many-body theory 21]. It lends itself naturally to the study of problems where an extra particle interacts with a closed-shell target. The use of diagrams makes this method

*E-mail: g.gribakin@am.qub.ac.uk

†E-mail: j.ludlow@am.qub.ac.uk 
both descriptive and intuitive, and allows one to take many-particle correlations into account in a systematic way. Many-body theory has been successful in the study of photoionisation 22], and in problems involving electrons, such as electron-atom scattering [23, 24, 25, 26], negative ions 27, 28, 29, 30], and precise calculations of energies and transition amplitudes in heavy atoms with a single valence electron [31, 32]. The application of many-body theory to low-energy positron interactions with atoms has met with more difficulty.

Many-body theory utilises techniques originating in quantum field theory. It describes the terms of the perturbation series in the interaction between particles diagrammatically. The difficulty in applying this approach to the interaction of positrons with atoms arises from the need to take into account (virtual) Ps formation. Being a bound state, Ps cannot be described by a finite number of perturbation-theory terms. Hence, an infinite sequence of the 'ladder' diagrams must be summed.

The first attempt to apply many-body theory to the positron-atom problem was by Amusia et al in 1976 [2], who used a crude approximate method of accounting for virtual Ps formation in He. A better approximation for the virtual Ps-formation contribution was devised in [4], and applications to various atomic targets, including noble gases, were reported [33, 34, 35]. In particular, a reasonable description was obtained for positron scattering from noble-gas atoms, which highlighted the presence of positron-atom virtual levels in $\mathrm{Ar}, \mathrm{Kr}$ and Xe. On the other hand, application of the same approximation to positron-atom annihilation showed that it was clearly deficient.

In spite of the approximate treatment of virtual Ps formation, many-body theory calculations for $\mathrm{Mg}, \mathrm{Cd}, \mathrm{Zn}$ and $\mathrm{Hg}$ were the first to provide credible evidence that positrons can bind to neutral atoms [33]. Two years later positron-atom binding was proved in a stochastic variational calculation for $\mathrm{Li}$ [36]. At present the list of atoms capable of binding positrons has expanded dramatically, SVM and configuration-interaction calculations confirming positron binding to $\mathrm{Mg}, \mathrm{Cd}$ and $\mathrm{Zn}$ [37, 38]. This topic is now of major interest in positron physics (see [38, 39] for useful reviews).

In this paper, new techniques will be outlined that allow the exact calculation of the electron-positron ladder diagram sequence which accounts for virtual Ps formation. This approach enables many-body theory to provide accurate information on the elastic scattering, annihilation and binding of positrons on atoms and negative ions at energies below the Ps formation threshold.

\section{MANY-BODY THEORY METHOD}

\section{A. Dyson equation}

A conventional treatment of positron scattering from an $N$-electron target would start from the Schrödinger equation for the total wavefunction for the $N+1$ particles. In many-body theory we start instead from the Dyson equation (see, e.g., [21, 40]),

$$
\left(H_{0}+\Sigma_{\varepsilon}\right) \psi_{\varepsilon}=\varepsilon \psi_{\varepsilon}
$$

where $\psi_{\varepsilon}$ is the single-particle (quasi-particle) wavefunction of the positron, $\varepsilon$ is its energy, and $H_{0}$ is a central-field Hamiltonian of the zeroth approximation, which describes the motion of the positron in the static field of the target. The many-body dynamics in Eq. (11) is represented by $\Sigma_{\varepsilon}$, a nonlocal energy-dependent correlation potential. This quantity, also known as the optical potential, is equal to the self-energy part of the single-particle Green's function of the positron in the presence of the atom [41]. Due to its nonlocal nature $\Sigma_{\varepsilon}$ operates on the quasi-particle wavefunction as an integral operator,

$$
\Sigma_{\varepsilon} \psi_{\varepsilon}=\int \Sigma_{\varepsilon}\left(\mathbf{r}, \mathbf{r}^{\prime}\right) \psi_{\varepsilon}\left(\mathbf{r}^{\prime}\right) d \mathbf{r}^{\prime}
$$

For hydrogen $H_{0}$ may simply be taken as the Hamiltonian of the positron moving in the electrostatic field of the ground-state atom, $H_{0}=-\frac{1}{2} \nabla^{2}+U(r)$, where $U(r)=\left(1+r^{-1}\right) e^{-2 r}$ [42] (we use atomic units throughout). For systems containing more than one electron the Hartree-Fock (HF) Hamiltonian (without exchange, for the positron) is the best choice. The correlation potential $\Sigma_{\varepsilon}$ is given by an infinite perturbation series in powers of the residual electron-electron and electron-positron interaction. Inclusion of the electrostatic interaction in $H_{0}$ and the use of the HF approximation for the target electrons means that the perturbation-theory expansion for $\Sigma_{\varepsilon}$ starts with the 2nd-order diagrams, and that the diagrams do not contain elements which describe the electrostatic potential [43].

Owing to the spherical symmetry of the problem, Eq. (1) can be solved separately for each positron partial wave. So, in practice one deals with radial quasiparticle wavefunctions, $\tilde{P}_{\varepsilon l}(r)$, related to $\psi_{\varepsilon}$ by $\psi_{\varepsilon}(\mathbf{r})=r^{-1} \tilde{P}_{\varepsilon l}(r) Y_{l m}(\Omega)$, where $Y_{l m}(\Omega)$ is the spherical harmonic for the orbital angular momentum $l$. Accordingly, the self-energy operator is also found for each partial wave separately, as $\Sigma_{\varepsilon}^{(l)}\left(r, r^{\prime}\right)$, see Eq. (A1) in the Appendix. 


\section{B. Correlation potential}

Figure 1 shows the lowest-order terms of the diagrammatic expansion for the correlation potential $\Sigma$, or more precisely, for the matrix element $\left\langle\varepsilon^{\prime}\left|\Sigma_{E}\right| \varepsilon\right\rangle$ of the correlation potential calculated at some energy $E$ between the positron states $\varepsilon$ and $\varepsilon^{\prime}$. The leading 2nd-order diagram, (a) in Fig. 1. corresponds to the following expression,

$$
\left\langle\varepsilon^{\prime}\left|\Sigma_{E}^{(2)}\right| \varepsilon\right\rangle=\sum_{\nu, \mu, n} \frac{\left\langle\varepsilon^{\prime} n|V| \mu \nu\right\rangle\langle\nu \mu|V| n \varepsilon\rangle}{E-\varepsilon_{\nu}-\varepsilon_{\mu}+\varepsilon_{n}+i 0},
$$

where $V$ is the electron-positron Coulomb interaction, the sum runs over all intermediate positron states $\nu$, excited electron states $\mu$ and hole states $n$, and $i 0$ is an infinitesimal positive imaginary quantity.

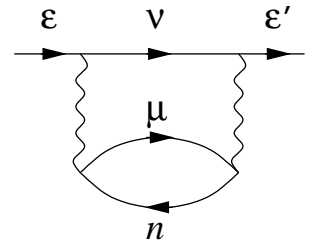

(a)

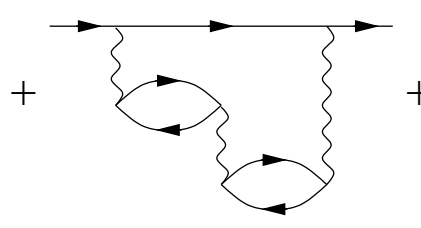

(e)

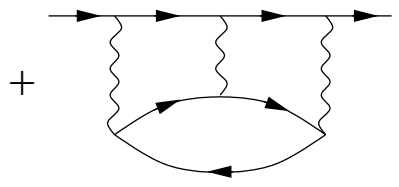

(b)

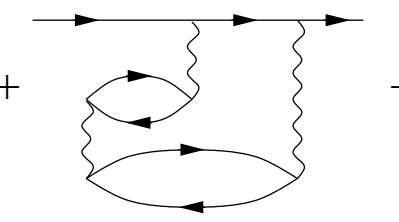

(f)

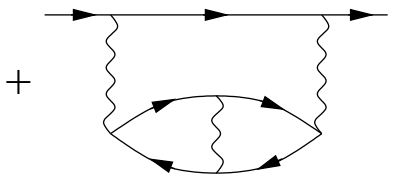

(c)

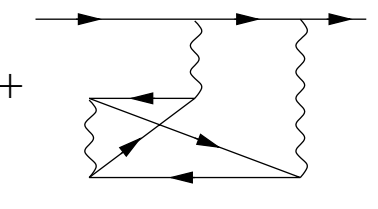

(g)

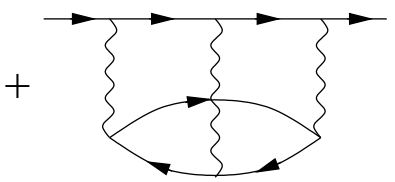

(d)

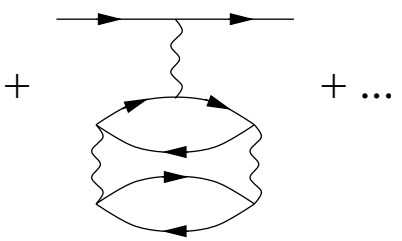

(h)

FIG. 1: Diagrammatic expansion of the positron-atom correlation potential $\Sigma$. Shown are the 2nd-order and main 3rd-order contributions. The top line in all the diagrams corresponds to the positron. Other lines with the arrows to the right describe excited electron states, while those with the arrows to the left correspond to holes, i.e., the electron states occupied in the atomic ground state. Wavy lines are the electron-positron or electron-electron Coulomb interactions.

It is easy to see from Eq. (3) that at low energies $E$ the diagonal matrix element $\left\langle\varepsilon\left|\Sigma_{E}^{(2)}\right| \varepsilon\right\rangle$ is negative. This means that the 2nd-order contribution to the correlation potential, Fig. 1(a), describes attraction. In fact, this diagram accounts for the main correlation effect in low-energy scattering, namely the polarisation of the atom by the charged projectile. At large distances it leads to a well-known local polarisation potential,

$$
\Sigma_{E}^{(2)}\left(\mathbf{r}, \mathbf{r}^{\prime}\right) \sim-\frac{\alpha}{2 r^{4}} \delta\left(\mathbf{r}-\mathbf{r}^{\prime}\right),
$$

where $\alpha$ is the static dipole polarisability of the atom in the HF approximation,

$$
\alpha=\frac{2}{3} \sum_{\mu, n} \frac{|\langle\mu|\mathbf{r}| n\rangle|^{2}}{\varepsilon_{\mu}-\varepsilon_{n}} .
$$

Besides the 2nd-order diagram, Fig. 1]shows the main 3rd-order contributions. A complete list of 3rd-order diagrams includes mirror images of the diagrams (f) and $(\mathrm{g})$. There are also a few more diagrams similar to diagram $(\mathrm{h})$, where the positron line is connected to the atomic excitation part by a single line. They represent correlation corrections to the HF electron charge density of the ground-state atom. Such corrections are much smaller than other correlations effects [32], and can be neglected. The total number of the 3rd-order diagrams in the positron-atom problem is considerably smaller than that in the electron case (see, e.g., [32]), where one needs to allow for the exchange between the incident and core electrons.

Comparing diagrams (c), (e), (f) and (g) with (a) in Fig. 1 we see that they represent corrections to the leading polarisation diagram, $\Sigma^{(2)}$, due to electron correlations within the atom. Interaction between electron-hole pairs can in principle be included in all orders, which would correspond to the random phase approximation (RPA) treatment of atomic polarisation [44]. On the other hand, if the two hole orbitals in diagrams (c) and (e) are identical, these diagrams together with similar higher-order terms, are easily incorporated within the 2nd-order diagram by calculating 
the excited electron states $\mu$ in the field of the atom with a hole in this orbital 24]. These approximations, and even the "bare" 2nd-order approximation (with exchange diagrams added in both cases), give good results in electron-atom scattering and negative ion problems [25, 26, 27, 28, 29, 30].

However, for the positron-atom problem the approximation based on diagrams (a) and corrections of types (c), (e), (f), and (g), proved to be deficient [2, 3, 4]. In addition one must include the diagram Fig. 1 (b) and higher-order diagrams in which the positron interacts with the excited electron in the intermediate state, Fig. 2] This sequence of diagrams accounts for virtual Ps formation. It is important that it is summed to all orders, since in quantum mechanics a bound state (here, Ps) which is absent in the zeroth approximation cannot be described by a finite number of perturbation theory terms.
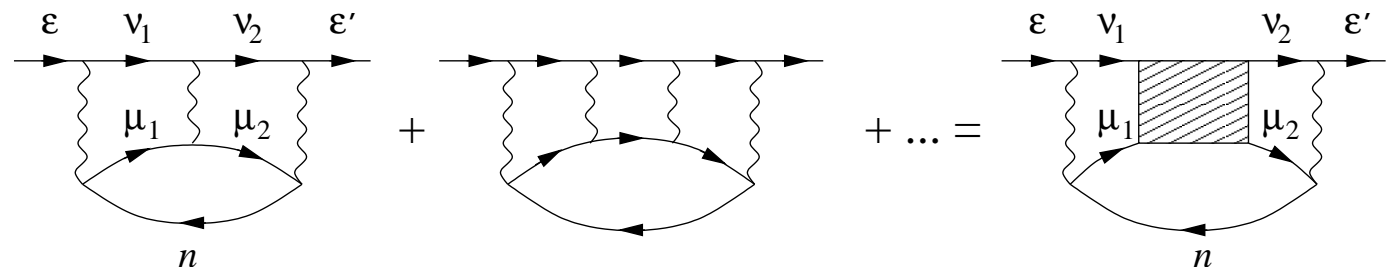

FIG. 2: Virtual Ps contribution to the positron-atom correlation potential $\Sigma$.

Summation of the diagrammatic sequence shown in Fig. 2 is done by calculating the electron-positron vertex function $\Gamma$, defined here as the sum of the electron-positron ladder diagrams, Fig. 3] and denoted in the diagram by the shaded block.

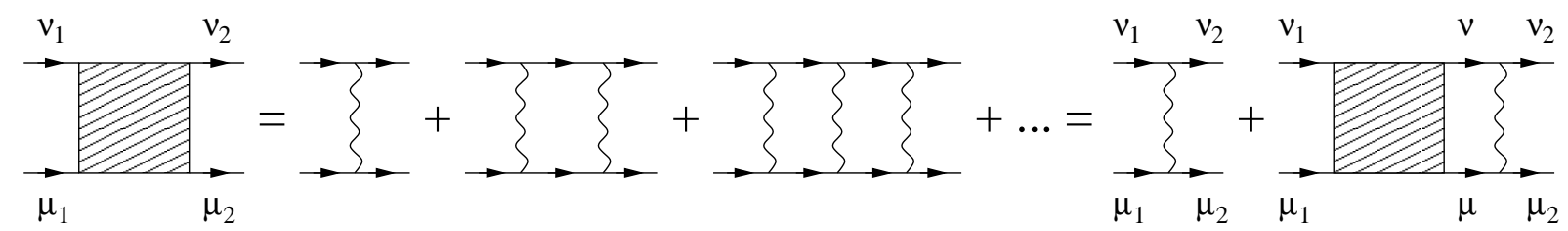

FIG. 3: Electron-positron ladder diagram sequence and its sum, the vertex function $\Gamma$.

Comparing the left- and right-hand sides of the diagrammatic equation in Fig. 3, we see that the vertex function satisfies the following linear equation,

$$
\left\langle\nu_{2} \mu_{2}\left|\Gamma_{E}\right| \mu_{1} \nu_{1}\right\rangle=\left\langle\nu_{2} \mu_{2}|V| \mu_{1} \nu_{1}\right\rangle+\sum_{\nu, \mu} \frac{\left\langle\nu_{2} \mu_{2}|V| \mu \nu\right\rangle\left\langle\nu \mu\left|\Gamma_{E}\right| \mu_{1} \nu_{1}\right\rangle}{E-\varepsilon_{\nu}-\varepsilon_{\mu}+i 0} .
$$

The vertex function depends on the energy $E$. It has the meaning of the electron-positron scattering amplitude in the field of the atom. In the lowest-order approximation $\Gamma_{E}=V$.

Once the vertex function is found, the contribution of virtual Ps to the correlation potential, Fig. 2] is obtained as

$$
\left\langle\varepsilon^{\prime}\left|\Sigma_{E}^{(\Gamma)}\right| \varepsilon\right\rangle=\sum_{\nu_{i}, \mu_{i}, n} \frac{\left\langle\varepsilon^{\prime} n|V| \mu_{2} \nu_{2}\right\rangle\left\langle\nu_{2} \mu_{2}\left|\Gamma_{E+\varepsilon_{n}}\right| \mu_{1} \nu_{1}\right\rangle\left\langle\nu_{1} \mu_{1}|V| n \varepsilon\right\rangle}{\left(E-\varepsilon_{\nu_{2}}-\varepsilon_{\mu_{2}}+\varepsilon_{n}+i 0\right)\left(E-\varepsilon_{\nu_{1}}-\varepsilon_{\mu_{1}}+\varepsilon_{n}+i 0\right)} .
$$

Since the electron-positron Coulomb interaction is attractive, $V<0$, the terms of the perturbation series in Fig. 3 have the same sign. This explains why their sum, and hence, the contribution of virtual Ps formation to the positronatom attraction, are large. Note that for electron scattering $(V>0)$ this series is alternating. As a result the net contribution of the diagrammatic series on the left-hand side in Fig. 2 is small, and its omission in the electron-atom correlation potential does not give rise to large errors.

The Ps-formation contribution to the correlation potential was previously approximated by using the free $1 s$-state Ps propagator orthogonalised to the ground-state electron wavefunctions [4, 34],

$$
\left\langle\varepsilon^{\prime}\left|\Sigma_{E}^{(\Gamma)}\right| \varepsilon\right\rangle \approx \sum_{n} \int \frac{\left\langle\varepsilon^{\prime} n|V| \tilde{\Psi}_{1 s, \mathbf{K}}\right\rangle\left\langle\tilde{\Psi}_{1 s, \mathbf{K}}|V| n \varepsilon\right\rangle}{E+\varepsilon_{n}-E_{1 s}+K^{2} / 4+i 0} \frac{d \mathbf{K}}{(2 \pi)^{3}},
$$

where $\Psi_{1 s, \mathbf{K}}=(8 \pi)^{-1 / 2} \exp \left(-\left|\mathbf{r}-\mathbf{r}^{\prime}\right| / 2\right) \exp \left[i \mathbf{K} \cdot\left(\mathbf{r}+\mathbf{r}^{\prime}\right) / 2\right]$ is the wave function of Ps $(1 s)$ with momentum $\mathbf{K}$, $E_{1 s}+K^{2} / 4$ is the energy of this state, and the tilde above $\Psi_{1 s, \mathbf{K}}$ in Eq. (8) indicates orthogonalisation. This 
approximation is suitable for positron scattering from the targets where ground-state Ps formation dominates, e.g., hydrogen or noble gas atoms. It also allows one to consider positron scattering above the Ps-formation threshold, where the correlation potential acquires an imaginary part due to the pole in the integral in Eq. (8) [35, 45]. At the same time, the ground-state Ps propagator fails to describe the short-range electron-positron correlations crucial for the calculation of the annihilation rates [34]. By the uncertainty principle, small separations invoke contributions of highly excited states of the Ps internal motion, not included in the $\operatorname{Ps}(1 s)$ propagator. In contrast, our present method based on the summation of the ladder diagram series, Eqs. (6) and (7), is consistent and complete. It accounts for all (virtual) intermediate states of the electron-positron pair.

For positron scattering on hydrogen, only a few types of diagrams contribute to $\Sigma_{E}$, since only one hole can be created. Moreover, the interaction of the intermediate-state electron and positron with the hole [diagrams (c) and (d) in Fig. [1 can be taken into account by calculating the intermediate electron and positron wavefunctions in the Coulomb field of the nucleus. In this case, the correlation potential is given by the sum of the 2nd-order diagram and the virtual Ps contribution, Figs. 1(a) and 2 and

$$
\Sigma_{E}=\Sigma_{E}^{(2)}+\Sigma_{E}^{(\Gamma)}
$$

is the exact correlation potential. In particular, the long-range asymptotic behaviour of $\Sigma_{E}^{(2)}$ at low energies, Eq. (4), contains the exact polarisability of hydrogen, $\alpha=\frac{9}{2}$.

\section{Scattering}

Rather than solving the Dyson equation for the quasiparticle wavefunction in the coordinate representation, it is easier to work with the self-energy matrix,

$$
\left\langle\varepsilon^{\prime}\left|\Sigma_{E}\right| \varepsilon\right\rangle=\int \varphi_{\varepsilon^{\prime}}^{*}(\mathbf{r}) \Sigma_{E}\left(\mathbf{r}, \mathbf{r}^{\prime}\right) \varphi_{\varepsilon}\left(\mathbf{r}^{\prime}\right) d \mathbf{r} d \mathbf{r}^{\prime}
$$

where $\varphi_{\varepsilon}$ are the positron eigenfunctions of the HF (or ground-state hydrogen) Hamiltonian $H_{0}$,

$$
H_{0} \varphi_{\varepsilon}=\varepsilon \varphi_{\varepsilon}
$$

with a given angular momentum $l, \varphi_{\varepsilon}(\mathbf{r})=r^{-1} P_{\varepsilon l}(r) Y_{l m}(\Omega)$. Since the static potential of the atom is repulsive, all positron states $\varphi_{\varepsilon}$ lie in the continuum $(\varepsilon>0)$. The radial wavefunctions are normalised to a $\delta$-function of energy in Rydberg, $\delta\left(k^{2}-k^{\prime 2}\right)$, where $k$ is the positron momentum. This corresponds to the asymptotic behaviour

$$
P_{\varepsilon l}(r) \sim(\pi k)^{-1 / 2} \sin \left(k r-l \pi / 2+\delta_{l}^{(0)}\right),
$$

where $\delta_{l}^{(0)}$ is the scattering phase shift in the static potential.

The matrix (10) can be used to obtain the phaseshifts directly [25]. First, a 'reducible' self-energy matrix $\left\langle\varepsilon^{\prime}\left|\tilde{\Sigma}_{E}\right| \varepsilon\right\rangle$ is found via the integral equation,

$$
\left\langle\varepsilon^{\prime}\left|\tilde{\Sigma}_{E}\right| \varepsilon\right\rangle=\left\langle\varepsilon^{\prime}\left|\Sigma_{E}\right| \varepsilon\right\rangle+\mathcal{P} \int \frac{\left\langle\varepsilon^{\prime}\left|\tilde{\Sigma}_{E}\right| \varepsilon^{\prime \prime}\right\rangle\left\langle\varepsilon^{\prime \prime}\left|\Sigma_{E}\right| \varepsilon\right\rangle}{E-\varepsilon^{\prime \prime}} d \varepsilon^{\prime \prime}
$$

where $\mathcal{P}$ means the principal value of the integral. The phase shift is then given by,

$$
\delta_{l}=\delta_{l}^{(0)}+\Delta \delta_{l}
$$

where

$$
\tan \Delta \delta_{l}=-2 \pi\left\langle\varepsilon\left|\tilde{\Sigma}_{\varepsilon}\right| \varepsilon\right\rangle
$$

determines the additional phaseshift $\Delta \delta_{l}(k)$ due to correlations, at the positron energy $\varepsilon$.

Once the reducible self-energy matrix has been found, the quasiparticle wavefunction (also known as the Dyson orbital) can be found via,

$$
\psi_{\varepsilon}(\mathbf{r})=\varphi_{\varepsilon}(\mathbf{r})+\mathcal{P} \int \varphi_{\varepsilon^{\prime}}(\mathbf{r}) \frac{\left\langle\varepsilon^{\prime}\left|\tilde{\Sigma}_{\varepsilon}\right| \varepsilon\right\rangle}{\varepsilon-\varepsilon^{\prime}} d \varepsilon^{\prime}
$$


In order to normalise the quasiparticle radial wavefunction at large distances to

$$
\tilde{P}_{\varepsilon l}(r) \sim(\pi k)^{-1 / 2} \sin \left(k r-l \pi / 2+\delta_{l}^{(0)}+\Delta \delta_{l}\right),
$$

the function obtained from the right-hand side of Eq. (16) must be multiplied by the factor,

$$
\cos \Delta \delta_{l}=\left[1+\left(2 \pi\left\langle\varepsilon\left|\tilde{\Sigma}_{\varepsilon}\right| \varepsilon\right\rangle\right)^{2}\right]^{-1 / 2} .
$$

\section{Positron annihilation}

The annihilation rate $\lambda$, of a positron in a gas of number density $n$ is usually expressed in terms of the effective number of electrons, $Z_{\text {eff }}$, which contribute to annihilation on an atom or molecule [46],

$$
\lambda=\pi r_{0}^{2} c n Z_{\mathrm{eff}},
$$

where $r_{0}$ is the classical electron radius and $c$ is the speed of light. Equation (19) defines $Z_{\text {eff }}$ as the ratio of the positron two-photon annihilation cross section of the atom to the spin-averaged two-photon annihilation cross section of a free electron in the Born approximation [47. Annihilation takes place at very small electron-positron separations, $\hbar /(m c) \sim 10^{-2}$ a.u. Hence, for nonrelativistic positrons it is determined by the electron density at the positron, and $Z_{\text {eff }}$ can be calculated as [46],

$$
Z_{\text {eff }}=\sum_{i=1}^{N} \int\left|\Psi\left(\mathbf{r}_{1}, \mathbf{r}_{2}, \ldots, \mathbf{r}_{N}, \mathbf{r}\right)\right|^{2} \delta\left(\mathbf{r}_{i}-\mathbf{r}\right) d \mathbf{r}_{1} \ldots d \mathbf{r}_{N} d \mathbf{r},
$$

where $\Psi\left(\mathbf{r}_{1}, \mathbf{r}_{2}, \ldots, \mathbf{r}_{N}, \mathbf{r}\right)$ is the full $(N+1)$-particle wavefunction of the $N$ electron coordinates $\mathbf{r}_{i}$ and positron coordinate $\mathbf{r}$. The wavefunction is normalised to a positron plane wave at large positron-atom separations,

$$
\Psi\left(\mathbf{r}_{1}, \mathbf{r}_{2}, \ldots, \mathbf{r}_{N}, \mathbf{r}\right) \simeq \Phi_{0}\left(\mathbf{r}_{1}, \mathbf{r}_{2}, \ldots, \mathbf{r}_{N}\right) e^{i \mathbf{k} \cdot \mathbf{r}},
$$

where $\Phi_{0}\left(\mathbf{r}_{1}, \mathbf{r}_{2}, \ldots, \mathbf{r}_{N}\right)$ is the atomic ground-state wavefunction, and $\mathbf{k}$ is the incident positron momentum.

Although $Z_{\text {eff }}$ is basically a cross section, Eq. (20) has the form of a transition amplitude. This enables one to apply the apparatus of many-body theory to this quantity directly [3, 34]. In this "transition amplitude" the initial and final states are identical, and the electron-positron two-body operator, $\sum_{i} \delta\left(\mathbf{r}_{i}-\mathbf{r}\right)$, plays the role of a perturbation. The positron energy in the initial and final states is the same, $\varepsilon=k^{2} / 2$, and (owing to the spherical symmetry of the target) the perturbation conserves the positron angular momentum $l$. Therefore, the contribution of each positron partial wave to $Z_{\text {eff }}$ can be determined separately. The corresponding many-body diagrammatic expansion

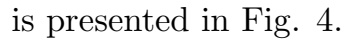

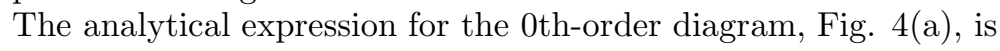

$$
Z_{\mathrm{eff}}^{(0)}=\sum_{n} \int \psi_{\varepsilon}^{*}(\mathbf{r}) \varphi_{n}^{*}\left(\mathbf{r}_{1}\right) \delta\left(\mathbf{r}-\mathbf{r}_{1}\right) \varphi_{n}\left(\mathbf{r}_{1}\right) \psi_{\varepsilon}(\mathbf{r}) d \mathbf{r} d \mathbf{r}_{1}=\sum_{n} \int\left|\varphi_{n}(\mathbf{r})\right|^{2}\left|\psi_{\varepsilon}(\mathbf{r})\right|^{2} d \mathbf{r},
$$

where $\psi_{\varepsilon}$ is the positron wavefunction, $\varphi_{n}$ is the wavefunction of the hole, and the sum over $n$ runs over all holes, i.e. orbitals occupied in the target ground state. This contribution is simply an overlap of the electron and positron densities, $\sum_{n}\left|\varphi_{n}(\mathbf{r})\right|^{2}$ and $\left|\psi_{\varepsilon}(\mathbf{r})\right|^{2}$, respectively.

The two 1st-order "corrections", Fig. 四(b) and (c), are identical, and their contribution is

$$
Z_{\mathrm{eff}}^{(1)}=2 \sum_{\nu, \mu, n} \frac{\langle\varepsilon n|\delta| \mu \nu\rangle\langle\nu \mu|V| n \varepsilon\rangle}{\varepsilon-\varepsilon_{\nu}-\varepsilon_{\mu}+\varepsilon_{n}},
$$

cf. Eq. (3) for the 2nd-order contribution to the correlation potential. In the calculations of annihilation we assume that the positron energy is below all other inelastic thresholds, hence, we have dropped $i 0$ in the energy denominator. Physically, the 1st-order diagram describes positron annihilation with an electron "pulled out" from the atom by the positron's Coulomb field. Calculations in Refs. 3, 34] showed that for noble-gas atoms the size of the 1st-order corrections is approximately equal to the 0th-order contribution. This means that higher-order terms must also be taken into account. 


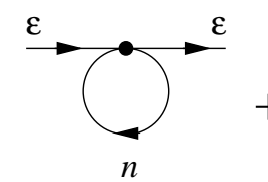

(a)

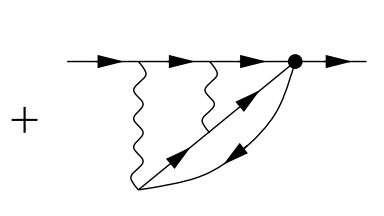

(e)

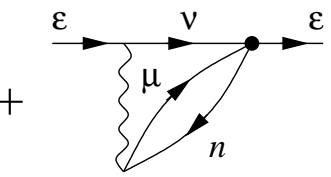

(b)

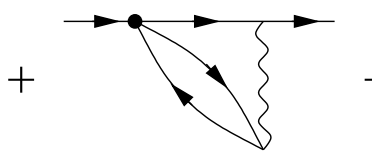

(c)

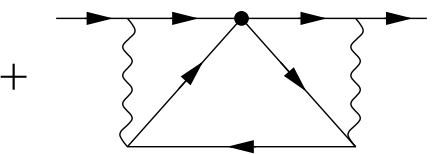

(d)

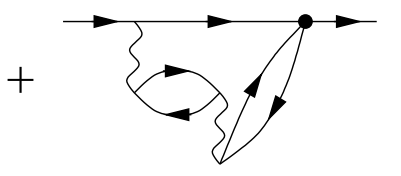

(f)

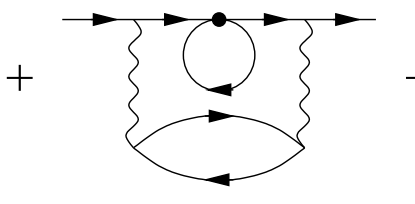

(g)

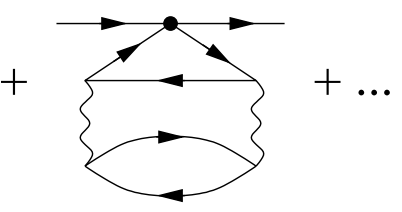

(h)

FIG. 4: Diagrammatic expansion of $Z_{\text {eff }}$. Apart from the 0th and 1st order diagrams (a), (b) and (c), the main types of 2nd-order diagrams are shown. The external lines of these diagrams $(\varepsilon)$ represent the wavefunction of the incident positron. The solid circle corresponds to the electron-positron $\delta$-function annihilation vertex.

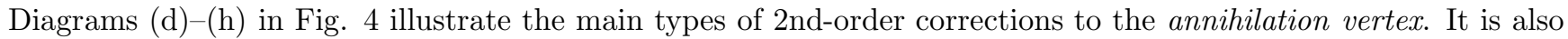
important to consider corrections to the incident positron wavefunctions denoted by $\varepsilon$. However, these corrections are included in all orders in the positron quasiparticle wavefunction, obtained from the Dyson equation (11), or via equation (16). Hence their contribution to $Z_{\text {eff }}$ is accounted for by using the positron Dyson orbitals $\psi_{\varepsilon}$ in the calculation of the annihilation diagrams.

The presence of the $\delta$-function operator in the annihilation diagrams enhances the importance of small electronpositron separations. For this reason, the most important diagrams in $Z_{\text {eff }}$ are those with the Coulomb interactions between the annihilating pair, e.g., the 2nd-order diagrams (d) and (e) in Fig. 团 (the latter together with its mirror image), and similar higher-order terms. A complete all-order calculation of their contribution is achieved by using the vertex function, as shown in Fig. [5] Note that for hydrogen this set of diagrams is exhaustive, provided the intermediate electron and positron states are calculated in the field of the bare nucleus. Previously diagrams (c)-(f) in Fig. [5 have only been estimated [34]. We will see that the ability to calculate these diagrams accurately is crucial for obtaining correct values of $Z_{\text {eff }}$. We will also see in Sec. IV that the role of the vertex function (representing virtual $\mathrm{Ps}$ ) in annihilation is much greater than in scattering.

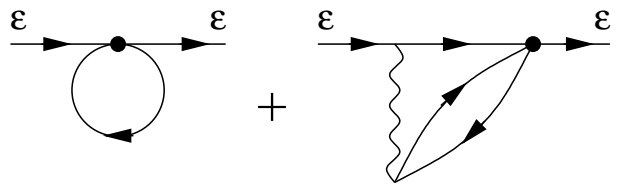

(a)

(b)

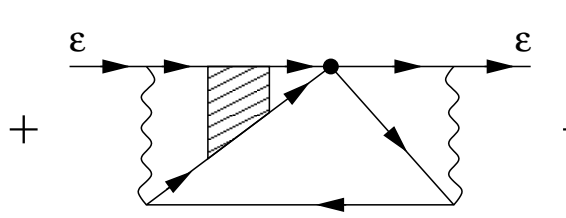

(e)

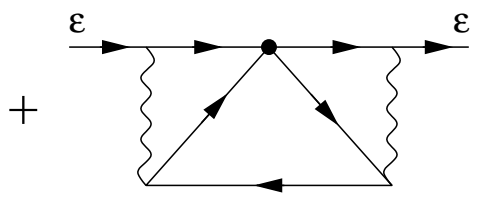

(c)

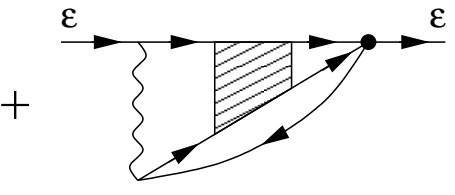

(d)

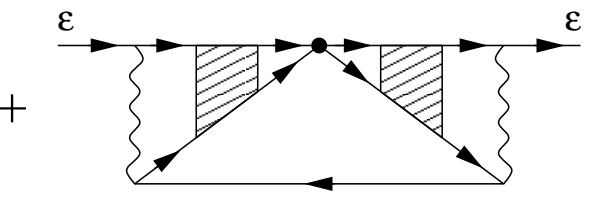

(f)

FIG. 5: Main contributions to the positron-atom annihilation parameter $Z_{\text {eff }}$. To account for the mirror images of the diagrams (b), (d) and (e), their contributions are multiplied by 2 .

Of course, for many-electron targets one can also consider other diagrams, e.g., (f)-(h) in Fig. 4 In particular, diagram (f) describes screening of the electron-positron Coulomb interaction by other electrons [cf. Fig. 1 (e)]. Diagram (g) can be viewed as the lowest-order "pick-off" annihilation contribution. Here the positron excites an electron-hole pair (a precursor of virtual Ps formation) and annihilates with an electron from one of the ground-state orbitals. Diagram (h) is independent of the positron energy. It represents one of the corrections to the HF ground-state 
electron density, cf. Fig. [(h). Unlike the diagrams in Fig. [5 these contributions are not systematically enhanced by the electron-positron Coulomb interaction at small distances.

It is clear from Fig. 4 that most correlation corrections to the annihilation vertex, including the dominant sequence of diagrams in Fig. [5 are nonlocal. As a result, the total $Z_{\text {eff }}$ can be written as

$$
Z_{\mathrm{eff}}=\int \sum_{n}\left|\varphi_{n}(\mathbf{r})\right|^{2}\left|\psi_{\varepsilon}(\mathbf{r})\right|^{2} d \mathbf{r}+\int \psi_{\varepsilon}^{*}(\mathbf{r}) \Delta_{\varepsilon}\left(\mathbf{r}, \mathbf{r}^{\prime}\right) \psi_{\varepsilon}\left(\mathbf{r}^{\prime}\right) d \mathbf{r} d \mathbf{r}^{\prime},
$$

where $\Delta_{\varepsilon}\left(\mathbf{r}, \mathbf{r}^{\prime}\right)$ represents the nonlocal correlation correction to the annihilation vertex. In the approximation of Fig. [5] it is equal to the sum of all diagrams (b)-(f) with the external positron lines $\varepsilon$ detached. Diagram (h) in Fig. [ and similar diagrams which represent corrections to the HF electron density, could be included by replacing of the HF electron density $\sum_{n}\left|\varphi_{n}(\mathbf{r})\right|^{2}$ in Eq. (24) with the exact target electron density $\rho_{e}(\mathbf{r})$. Given the high accuracy of the HF density, this would make only a small change in $Z_{\text {eff }}$. The structure of Eq. (24) shows that even when one uses the best single-particle positron wavefunction, the annihilation rate is not reduced to a simple (local) overlap of the electron and positron densities.

There is an important physical difference between the correlation effects in positron scattering and annihilation. The key role played by the long-range polarisation potential for low-energy positrons means that large distances are important. Polarisation also emphasises the role played by the dipole part of the positron-target Coulomb interaction and dipole target excitations. The contribution of virtual Ps formation to $\Sigma_{E}$ is a short-range effect. The typical distances here are comparable to the radius of the atom, or the radius of ground-state Ps. The net effect of the strong positron-atom attraction brings about low-lying virtual $s$-states (see, e.g., 42]) for $\mathrm{Ar}, \mathrm{Kr}$ and $\mathrm{Xe}$ [34], or positron-atom weakly bound states, e.g., in $\mathrm{Mg}$ [33, 35]. In both cases, the positron scattering phaseshifts and the positron quasiparticle wavefunction in the vicinity of the atom vary rapidly as functions of the positron energy.

On the contrary, in the annihilation diagrams the $\delta$-function vertex emphasises small electron-positron separations. By the uncertainty principle, such small separations correspond to high-energy excitations in the intermediate states in the diagrams (b)-(f), Fig. 5 As a result, the non-local correction to the annihilation vertex, $\Delta_{\varepsilon}$, has a weak energy dependence, and the energy dependence of the 2nd term in Eq. (24) is almost entirely due to that of the positron wavefunctions. The only exception is when the positron energy approaches the Ps-formation threshold from below. Here the virtual-Ps contribution, Fig. 5(f), rises sharply. Details of the threshold behaviour of $Z_{\text {eff }}$ are discussed in Ref. [48].

The large difference in the energy scales characteristic of positron scattering and annihilation has another physically important consequence. It turns out that the relative size of the annihilation vertex corrections in $Z_{\text {eff }}$, i.e. the ratio of the 2 nd term in Eq. (24) to $Z_{\text {eff }}^{(0)}$, is about the same, whether $\psi_{\varepsilon}$ are the positron wavefunctions in the repulsive static potential, $\psi_{\varepsilon}=\varphi_{\varepsilon}$, or the Dyson orbitals which fully account for the positron-atom correlation potential. Numerical illustrations of this effect will be provided in Sec. IV

Finally, we should mention that the correct normalisation of $Z_{\text {eff }}$ for positrons with angular momentum $l$ is obtained by multiplying the diagrams in Figs. 4 and 5 by the extra numerical factor,

$$
\frac{4 \pi^{2}}{k}(2 l+1)
$$

This follows from the structure of the positron wavefunction $\psi_{\mathbf{k}}$, which has the asymptotic behaviour of a plane wave $e^{i \mathbf{k} \cdot \mathbf{r}}$ at large distances [cf. Eq. [21)],

$$
\psi_{\mathbf{k}}=\frac{4 \pi}{r} \sqrt{\frac{\pi}{k}} \sum_{l=0}^{\infty} \sum_{m=-l}^{m=l} i^{l} e^{i \delta_{l}} \tilde{P}_{\varepsilon l}(r) Y_{l m}^{*}\left(\Omega_{\mathbf{k}}\right) Y_{l m}\left(\Omega_{\mathbf{r}}\right) .
$$

To derive Eq. 25), one can use $\psi_{\mathbf{k}}$ as the external positron lines in an annihilation diagram, and perform averaging over the directions of $\mathbf{k}$.

\section{NUMERICAL IMPLEMENTATION}

\section{A. Use of B-splines and convergence}

To evaluate the diagrams of the correlation potential $\Sigma$ and annihilation parameter $Z_{\text {eff }}$, one first needs to generate sets of electron and positron HF basis states. These are then used to calculate matrix elements of the Coulomb and $\delta$-function operators, the main building blocks of the diagrams. Evaluation of the diagrams requires summation 
over complete sets of electron and positron intermediate states, including integration over the electron and positron continua.

To perform a numerical calculation, the continuous spectrum can be discretised. The simplest way of doing this is by placing the system in a spherical cavity of radius $R$. Setting the wavefunctions to zero at the boundary will result in a discrete spectrum of eigenstates with an approximately constant stepsize in momentum space,

$$
\Delta k \approx \pi / R \text {. }
$$

If the value of $R$ is sufficiently large $\left(R \gg R_{\text {at }}\right.$, where $R_{\text {at }} \sim 1$ a.u. is the size of the atom), the presence of the boundary will not affect the quantities calculated. Indeed, for the positron energy below all inelastic thresholds (except, of course, annihilation), the intermediate states in the diagrams are virtual, and no particle in an intermediate state can escape to infinity.

The drawback of this procedure is that for a suitably large $R$, the stepsize in momentum is small, e.g., for $R=30$ a.u., $\Delta k \approx 0.1$ a.u. Hence, one would need large numbers of intermediate states to achieve convergence. Note that the actual upper energy limit depends on the quantity in question. Thus, diagrams in $Z_{\text {eff }}$ converge more slowly than those of the correlation potential $\Sigma$, because of the greater role of small electron-positron separations and high orbital angular momenta in the former. However, as a rough guide, summing up to the energy of $10^{2}$ a.u. should be sufficient for both. The question of the number of intermediate states is especially important for the calculation of the vertex function $\Gamma$, which is a $N_{\Gamma} \times N_{\Gamma}$ matrix [see Eq. [6] ], where $N_{\Gamma} \sim N^{2}\left(l_{\max }+1\right), N$ being the number of electron or positron states in each partial wave and $l_{\max }$ being the largest orbital angular momentum included. It is clear that here the simple cavity quantisation cannot work.

Instead, to achieve an accurate and economical span of the continuum we use B-splines [49]. B-splines of order $k$ are $n$ piecewize polynomials of degree $k-1$ defined by a knot sequence $r_{j}$ which divides the interval $[0, R]$ into $n-k+1$ segments [50]. The basis states are obtained by expanding the radial wavefunctions $P_{l}(r)$ in terms of B-splines $B_{i}(r)$,

$$
P_{l}(r)=\sum C_{i}^{(l)} B_{i}(r)
$$

and finding the eigenvectors and eigenvalues of the radial part of the HF (or hydrogen atom) Hamiltonian for each orbital angular momentum $l$ by solving the generalised eigenvalue problem,

$$
\sum H_{i j} C_{j}^{(l)}=\varepsilon \sum Q_{i j} C_{j}^{(l)},
$$

where $H_{i j}=\left\langle B_{i}\left|H_{0}^{(l)}\right| B_{j}\right\rangle$, and $Q_{i j}=\left\langle B_{i} \mid B_{j}\right\rangle$. Prior to solving Eq. (29), the ground-state atom HF Hamiltonian is generated by a conventional HF routine [51]. Note that in the sums over $i$ and $j$ in Eqs. (28) and (29) the first and last splines are discarded to implement the boundary condition $P_{l}(0)=P_{l}(R)=0$, leaving one with a set of $n-2$ eigenstates for each electron and positron orbital angular momentum. When Eq. (29) is solved for the electron, it yields the wavefunctions of the orbitals occupied in the atomic ground state (holes), as well as those of the excited states (particles). The exact energies of the excited electron and positron states are determined by the B-spline radial knot sequence.

It is instructive to try to design an ideal distribution of energies of a discrete set spanning the continuum. Qualitatively, at low energies $(\varepsilon \ll 1$ a.u.) the continuous spectrum states oscillate slowly, and the contribution of large distances in the matrix elements is important (hence, the need for a large $R$ ). As the energy of the states increases, the range of important distances becomes smaller and smaller. Indeed, the matrix elements then contain rapidly oscillating factors of $e^{i k r}$ type, which means that the dominant contribution comes from $r \lesssim k^{-1}$. Therefore, one does not need a large value of the cavity radius $R$ for the higher-energy states. More specifically, one can estimate the necessary radius as $R \sim a / k$, where $a$ is a number greater than unity. Combining this with the cavity quantisation condition (27), one obtains $\Delta k / k \sim \pi / a$, which yields the following grid in momentum space:

$$
k_{j}=k_{0} e^{\beta j},
$$

where $k_{0}$ is the lowest momentum, $\beta=\pi / a$, and $j=0,1,2, \ldots$ Thus, it appears that the optimal momentum and energy grids are exponential. By choosing a small initial momentum $k_{0} \ll 1$ a.u. and $\beta<1$ one ensures that the stepsize in momentum, $\Delta k \approx \beta k$, is sufficiently small, to describe accurately the energy variation of the quantities summed.

It turns out that basis sets generated by Eqs. (28) and (29) using an exponential radial knot sequence [49] are a very close realisation of the exponential energy grid, and are effectively complete. In the present work we use $n=40$ $\mathrm{B}$-splines of order $k=6$ with a knot sequence

$$
r_{j}=\rho\left(e^{\sigma j}-1\right)
$$


where $\rho=10^{-3}$ a.u., and $\sigma$ is determined by the condition $r_{n-k+1}=R$. Figure 6 shows the positive eigenenergies of the electron and positron basis states with $l=0$ for the hydrogen atom. Their distribution does indeed correspond to the exponential ansatz, Eq. (30), with $\beta \approx \sigma$. The highest energies in the sets are about $10^{8}$ a.u. This value is close to the magnitude of $(\rho \sigma)^{-2}$, since the knot point closest to the origin determines the most rapidly varying eigenstate (by the uncertainty principle). In fact, it may not be necessary to include all 38 basis states in each partial wave in the many-body theory sums. In the calculations reported in this paper we use only about 15 lowest states, which span the energy range from threshold to $\sim 10^{2}$ a.u.

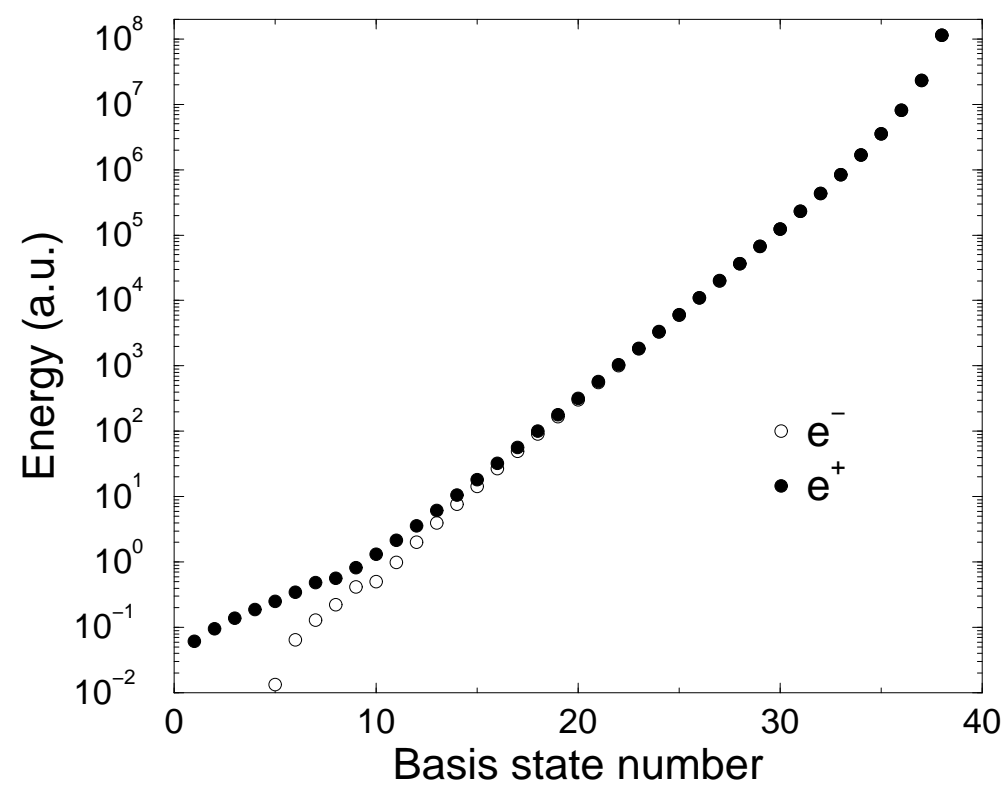

FIG. 6: Energies of the electron (open circles) and positron (solid circle) $s$-wave B-spline basis states in the field of the hydrogen nucleus, obtained using $R=30, k=6$ and $n=40$. The first four electron states with negative energies $(-0.50000,-0.12500$, -0.05542 and -0.00246 a.u.) are not shown.

Note that B-spline basis sets are used widely in atomic physics [49], and that there are other basis sets which show a near-exponential spanning of the continuum. In particular, Laguerre basis states provide rapid convergence in close-coupling electron-atom scattering calculations [52].

\section{B. Calculation of the self-energy and annihilation diagrams}

The self-energy and annihilation diagrams are calculated by summation over the B-spline basis states, and the vertex function is found by matrix inversion from Eq. (6). The angular parts of the states are separated in the matrix elements and the angular variables are integrated over analytically. The actual expressions for the diagrams are given in the Appendix. The self-energy matrix and the vertex function are energy dependent. In practice, the self-energy matrix has been calculated at 8 energies spaced evenly from zero to the Ps formation threshold. Interpolation onto any required energy $E$ is then used.

Apart from the B-spline basis states, we also consider true positron continuum HF states (12). They are needed to evaluate the matrix elements $\left\langle\varepsilon\left|\Sigma_{E}\right| \varepsilon^{\prime}\right\rangle$ and obtain the phaseshifts via Eqs. (13)-(15). Here we use 201 states that form an equidistant mesh in positron momenta of size $\Delta k=0.02$. A transformation of the B-spline basis matrix elements $\left\langle i\left|\Sigma_{E}\right| j\right\rangle$ into $\left\langle\varepsilon\left|\Sigma_{E}\right| \varepsilon^{\prime}\right\rangle$ could be done using the effective completeness of the B-spline states on the interval $[0, R]$

$$
\left\langle\varepsilon\left|\Sigma_{E}\right| \varepsilon^{\prime}\right\rangle=\sum_{i, j}\langle\varepsilon \mid i\rangle\left\langle i\left|\Sigma_{E}\right| j\right\rangle\left\langle j \mid \varepsilon^{\prime}\right\rangle,
$$

where $\langle\varepsilon \mid i\rangle$ is the overlap of the HF state with the B-spline basis state. However, unlike the B-spline states which satisfy the zero boundary condition at $r=R$, the continuous spectrum state $P_{\varepsilon l}$ is finite at the boundary. To fix this 
problem we insert a radial weighting function $f(r)=R-r$ into equation (32), which now reads

$$
\left\langle\varepsilon\left|\Sigma_{E}\right| \varepsilon^{\prime}\right\rangle=\sum_{i, j}\langle\varepsilon|f| i\rangle\left\langle i\left|f^{-1} \Sigma_{E} f^{-1}\right| j\right\rangle\left\langle j|f| \varepsilon^{\prime}\right\rangle
$$

and calculate the "weighted" self-energy matrix $\left\langle i\left|f^{-1} \Sigma_{E} f^{-1}\right| j\right\rangle$, rather than $\left\langle i\left|\Sigma_{E}\right| j\right\rangle$. The singularity of $f^{-1}$ at $r=R$ does not cause a problem, since the B-spline basis states in the Coulomb matrix elements involved, vanish at $r=R$. The same trick is applied in the calculation of the annihilation diagrams.

To calculate $\left\langle\varepsilon\left|\Sigma_{E}\right| \varepsilon^{\prime}\right\rangle$ more accurately at low positron energies, where distances beyond $r=R$ can be important, we make use of the long-range asymptotic form of the correlation potential (4). The contribution of $r>R$ can be evaluated as

$$
\int_{R}^{\infty} P_{\varepsilon l}(r)\left(-\frac{\alpha}{2 r^{4}}\right) P_{\varepsilon^{\prime} l}(r) d r,
$$

with the correct value of the dipole polarisability $\alpha$, and added to $\left\langle\varepsilon\left|\Sigma_{E}\right| \varepsilon^{\prime}\right\rangle$.

\section{Convergence with respect to the orbital angular momenta}

The use of a B-spline basis means that fast convergence is achieved with respect to the number of states with a particular orbital angular momentum. However, this leaves open the question of convergence with respect to the maximal orbital angular momentum of the electron and positron intermediate states included in the calculation. It has been known for a while that calculations of positron-atom scattering converge slowly with respect to the number of target angular momenta included in the expansion of the total wavefunction, notably slower than in the electronatom case [53]. This is also true for the configuration-interaction-type calculations of positron-atom bound states 17, 19, 54] and scattering [20]. Calculations of annihilation rates converge even more slowly [19, 20, 54]. Physically, the slow convergence rate arises from the need to describe virtual Ps localised outside the atom by an expansion in terms of single-particle orbitals centred on the nucleus.

The problem of the convergence rate with respect to the maximal orbital angular momentum has been investigated by the authors in Ref. [55]. Using a perturbation-theory approach and the original ideas of Schwartz [56], we derived asymptotic formulae that describe the convergence of the scattering amplitudes, or the phase shifts, and annihilation rates, or $Z_{\text {eff }}$. The contribution of high orbital angular momenta probes small particle separations in the system. The difference between the convergence rates of the scattering and annihilation parameters is due to the presence of either the Coulomb interaction or the $\delta$-function annihilation operator in the relevant amplitudes.

The increments to the phaseshifts and $Z_{\text {eff }}$ upon increasing the maximum orbital angular momentum from $\lambda-1$ to $\lambda$, were found to behave as $(\lambda+1 / 2)^{-4}$ and $(\lambda+1 / 2)^{-2}$, respectively. This means that if a series of calculations is stopped at some maximal angular momentum $\lambda=l_{\max }$, the values obtained approach the ultimate $\left(l_{\max } \rightarrow \infty\right)$ values as follows:

$$
\begin{gathered}
\delta_{l}^{\left[l_{\max }\right]} \simeq \delta_{l}-\frac{A}{\left(l_{\max }+1 / 2\right)^{3}}, \\
Z_{\mathrm{eff}}^{\left[l_{\max }\right]} \simeq Z_{\mathrm{eff}}-\frac{B}{\left(l_{\max }+1 / 2\right)} .
\end{gathered}
$$

where $A$ and $B$ are some constants. They are determined together with $\delta_{l}$ and $Z_{\text {eff }}$ by fitting Eqs. (35) and (36) to the numerical data obtained for a range of $l_{\max }$. This extrapolation to $l_{\max } \rightarrow \infty$ is performed at each positron momentum value considered, and is especially important for obtaining correct values of $Z_{\text {eff }}$.

\section{RESULTS: SCATTERING AND ANNIHILATION ON HYDROGEN}

The theory outlined above can be readily applied to any closed-shell atom or ion. In this paper we would like to test it for the simplest possible target, the hydrogen atom. Since it contains only one electron, the correlation potential from Eq. (9) and the $Z_{\text {eff }}$ diagrams in Fig. 5 give an exact solution of the elastic scattering and annihilation problems, provided the intermediate electron and positron states are calculated in the field of $\mathrm{H}^{+}$. The key advance of the present many-body theory of positron-atom interactions relates to the calculation of the electron-positron vertex 
function $\Gamma$, Eq. (6) and its incorporation in the self-energy and annihilation diagrams. It is mainly these features of the theory that a positron-hydrogen calculation is intended to test.

In the numerical implementation we first generate the electron and positron B-spline basis sets. They are then used to evaluate the matrix elements, find the vertex function and calculate the self-energy and annihilation diagrams (see Appendix). Using the self-energy matrix, the phaseshifts are obtained by means of Eqs. (13)-(15), and the positron Dyson orbital is calculated from Eqs. (16) and (18). In the end, the Dyson orbitals replace the positron HF states in the external lines of the annihilation diagrams, and final values of $Z_{\text {eff }}$ are obtained. To test the stability of the results with respect to the cavity radius, the calculations were performed with $R=15$ and 30 a.u.

To extrapolate the scattering phaseshifts and $Z_{\text {eff }}$ to $l_{\max } \rightarrow \infty$, as per Eqs. (35) and (36), the diagrams are evaluated for a range of maximal orbital angular momenta, $l_{\max }=7-10$. This procedure is illustrated by Fig. 7 for the phaseshifts and Fig. 8 for $Z_{\text {eff }}$, for the $s^{-}, p$ - and $d$-wave incident positron with momenta $k=0.2,0.4$, and 0.6 a.u.

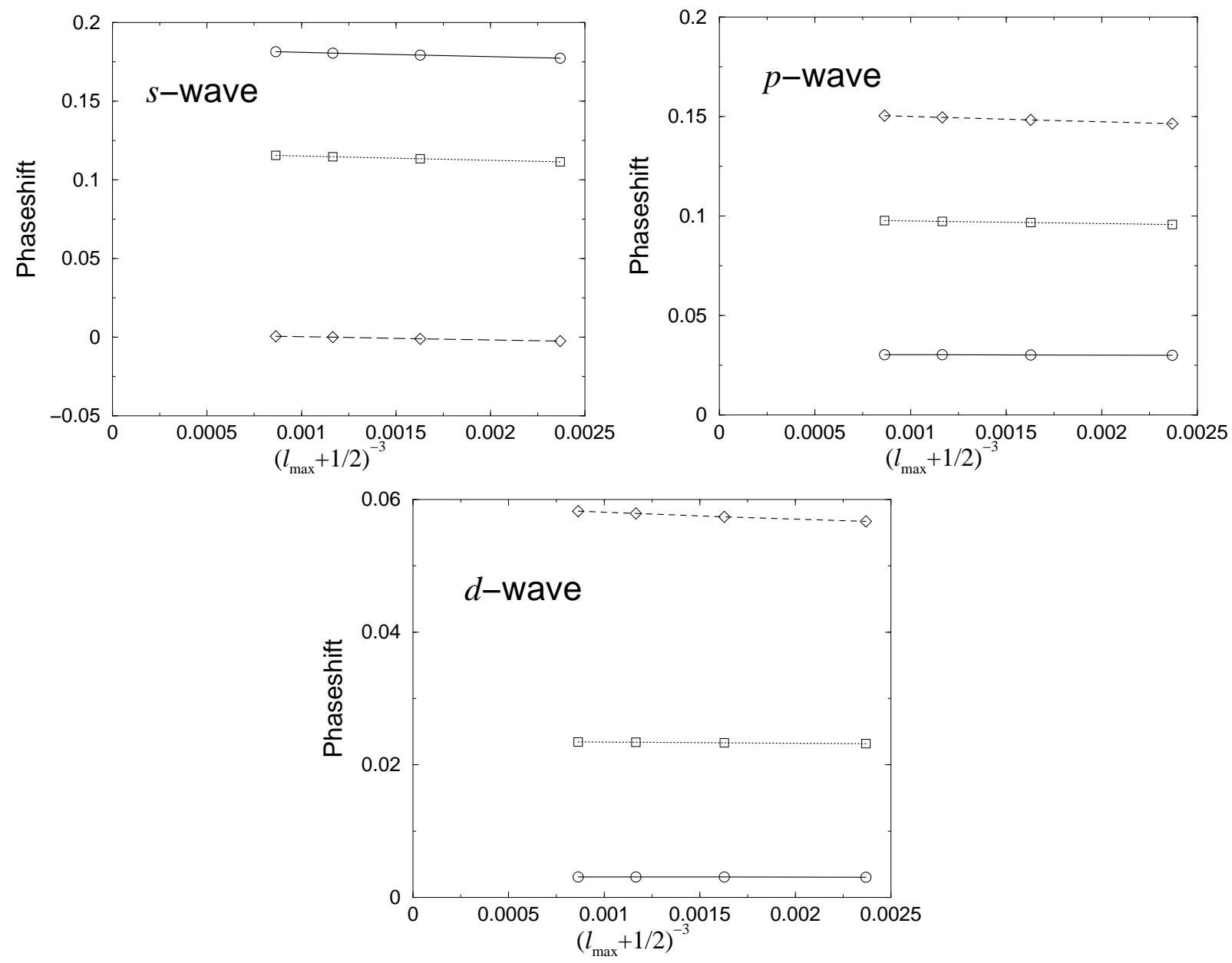

FIG. 7: Convergence of the $s^{-}, p$ - and $d$-wave positron scattering phaseshifts on hydrogen with respect to the maximal orbital angular momentum $l_{\max }$ for $R=15$ a.u. Circles, $k=0.2$ a.u.; squares, $k=0.4$ a.u.; diamonds, $k=0.6$ a.u.

Figures 7 and 8 show that the calculations have converged to the regime in which the asymptotic formulae (35) for $\delta_{l}$ and (36) for $Z_{\text {eff }}$, may be applied. The graphs also illustrate the point that the inclusion of high orbital angular momenta and extrapolation to $l_{\max } \rightarrow \infty$ is much more important in the calculations of annihilation, compared with scattering. For the positron momenta and partial waves shown, between $15 \%$ and $30 \%$ of the final value of $Z_{\text {eff }}$ is due to such extrapolation. Quantitatively, this contribution can be characterised by the ratio $B / Z_{\text {eff }}$, see Eq. (36), given in Table 【 Its increase with the positron angular momentum may be related to the greater role of the correlation corrections to the annihilation vertex in higher positron partial partial waves (see below).

Figure 9 shows the $s-, p$ - and $d$-wave phaseshifts for the total correlation potential (9). They are in very good agreement with those from an accurate variational calculation (see Ref. [13]), the discrepancy being of order $10^{-3} \mathrm{rad}$. The values obtained with $R=15$ and $R=30$ a.u. are almost indistinguishable, except at low positron momenta. Here the results for $R=30$ are superior to those for $R=15$. The larger cavity size allows for a better account of the 

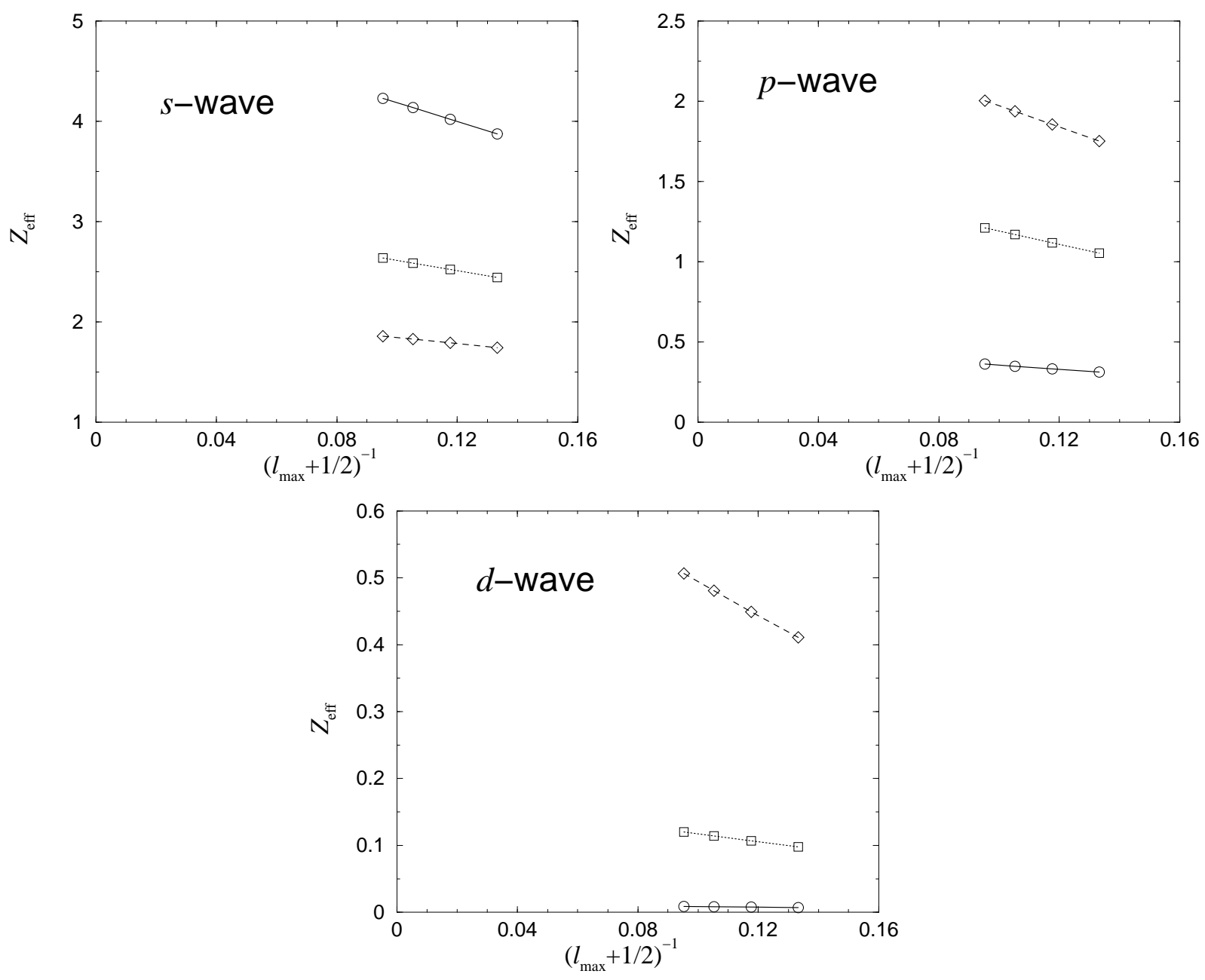

FIG. 8: Convergence of the $s^{-}, p$ - and $d$-wave contributions to $Z_{\text {eff }}$ for positron annihilation on hydrogen with respect to the maximal orbital angular momentum $l_{\max }$ for $R=15$ a.u. Circles, $k=0.2$ a.u.; squares, $k=0.4$ a.u.; diamonds, $k=0.6$ a.u.

TABLE I: Values of $B / Z_{\text {eff }}$ which characterise the dependence of calculated $Z_{\text {eff }}$ on $l_{\max }$.

\begin{tabular}{cccr}
\hline \hline $\begin{array}{c}\text { Momentum } \\
\text { (a.u.) }\end{array}$ & & Partial wave & \\
& $s$ & 2 & 3.33 \\
0.2 & 1.82 & 2.63 & 3.32 \\
0.4 & 1.62 & 2.58 & 3.37 \\
0.6 & 1.41 & 2.51 & 3. \\
\hline \hline
\end{tabular}

long range $-\alpha / 2 r^{4}$ tail in the polarisation potential.

Examining the phaseshifts allows us to compare the relative sizes of the polarisation and Ps-formation contributions to the correlation potential (9). The static positron-atom potential is repulsive, resulting in negative values of the phase shifts (thin solid curves). The inclusion of $\Sigma$, i.e., correlations, makes the low-energy phase shifts positive. Dashed curves in Fig. 9 show the phaseshifts obtained by including only the 2nd-order diagram $\Sigma^{(2)}$ (polarisation), while dot-dashed curves are those obtained with $\Sigma^{(\Gamma)}$ alone (virtual Ps-formation). We see that none of these results is close to the phaseshift obtained with the full $\Sigma$. This means that neither contribution dominates the correlation potential, and the inclusion of both polarisation and virtual Ps-formation effects is essential for solving the positronatom problem. Of course, any calculation which produces accurate positron-hydrogen phaseshifts contains these contributions implicitly. The advantage of the many-body theory approach is that one can separate them, and get a better insight into the physics of the system. 

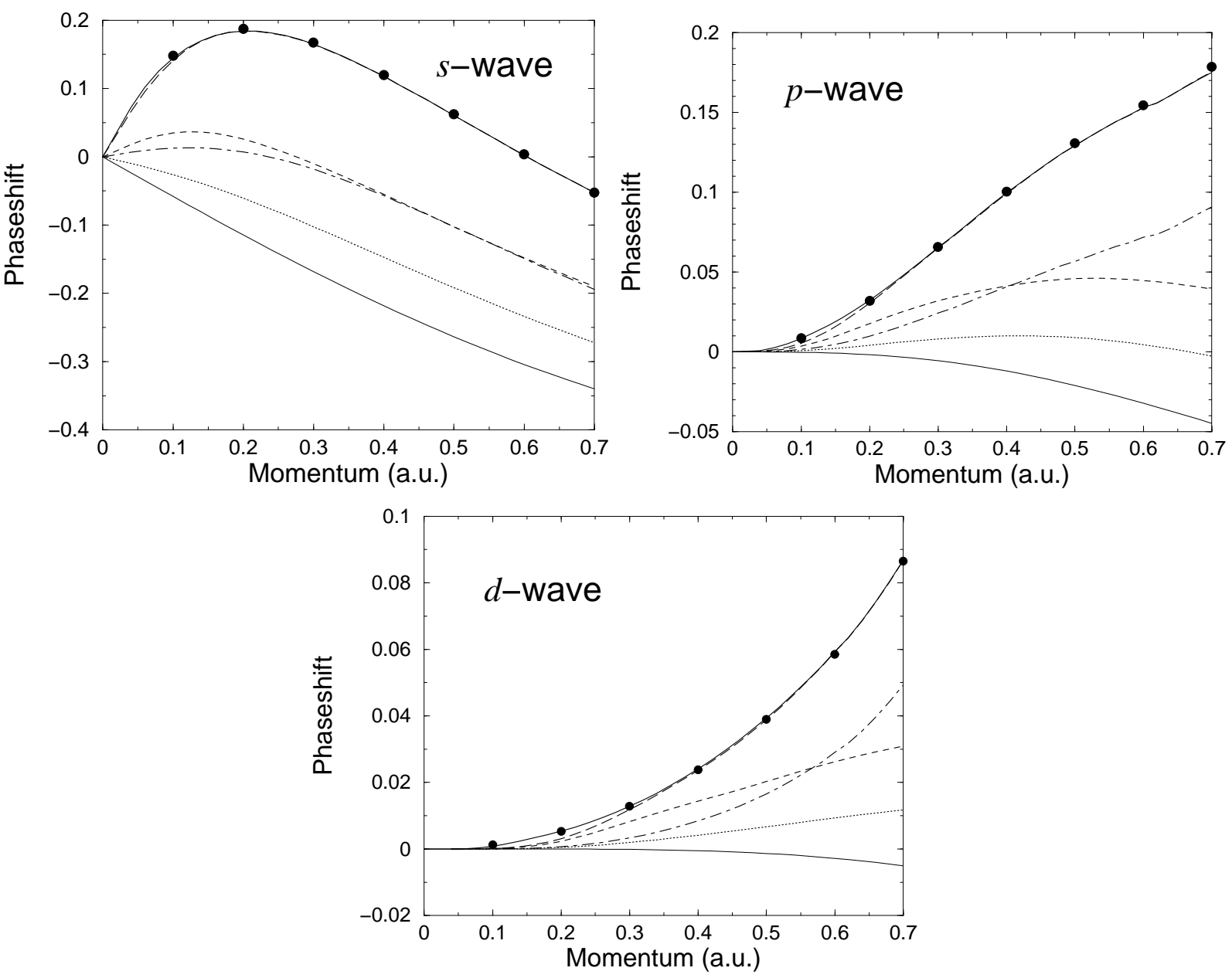

FIG. 9: Positron-hydrogen $s, p$ and $d$-wave scattering phaseshifts: long-dashed curve, many-body theory $(R=15$ a.u.); solid curve, many-body theory $(R=30$ a.u. $)$; circles, variational calculation [13]. Thin solid curve, static approximation; dashed curve, $\Sigma^{(2)}$; dot-dashed curve, $\Sigma^{(\Gamma)}$; dotted curve, $\Sigma^{(\Gamma)}$ obtained with $\Gamma=V($ all $R=15)$.

To illustrate the non-perturbative nature of virtual Ps formation we have also performed calculations that include the vertex function only to 1st order, $\Gamma_{E}=V$, in $\Sigma^{(\Gamma)}$ (dotted curves in Fig. 9). This approximation accounts only for about $50 \%$ of the total vertex function contribution. Note that the higher-order terms in $\Gamma$ become even more important close to the Ps formation threshold $(k \approx 0.7)$ in $p$ and $d$ waves. This is related to the virtual Ps becoming more "real" close to the threshold.

We now turn to positron annihilation. Having solved the scattering problem accurately with the full $\Sigma$, we are now in possession of the best (quasiparticle) positron wavefunction, the Dyson orbital. Before using it in all annihilation diagrams, let us first look at the effect of the Dyson orbital on the 0th-order diagram, Fig. [5(a), for the $s$-wave $Z_{\text {eff. }}$. Figure 10 shows that the 0th-order contribution, Eq. (22), evaluated with the positron wavefunction in the static atomic potential gives values up to 20 times smaller than the accurate variational results [57]. This situation is similar to that in positron annihilation on noble-gas atoms, where Eq. (22) evaluated with the static (HF) positron wavefunction underestimates experimental $Z_{\text {eff }}$ by a factor of $10^{1}-10^{3}$ at low positron energies [3, 34]. It is natural that the use of the Dyson orbital, which is "aware" of the positron-atom attraction, in Eq. (22), leads to increased $Z_{\text {eff }}$, and introduces a correct momentum dependence (dashed curve in Fig. 10). This latter fact is in agreement with the general understanding of the origins of the energy dependence and enhancement of $Z_{\text {eff }}$ at low energies, and their relation to positron-atom virtual states $[3,34,58]$. However, the absolute values obtained are still about 5 times lower than the benchmark.

The remaining $80 \%$ come from the nonlocal corrections to the annihilation vertex, diagrams (b)-(f) in Fig. [5 The contributions of all the diagrams evaluated using the positron Dyson orbitals, and the total $Z_{\text {eff }}$ are shown in Fig. [1] for the positron $s, p$ and $d$ partial waves. 


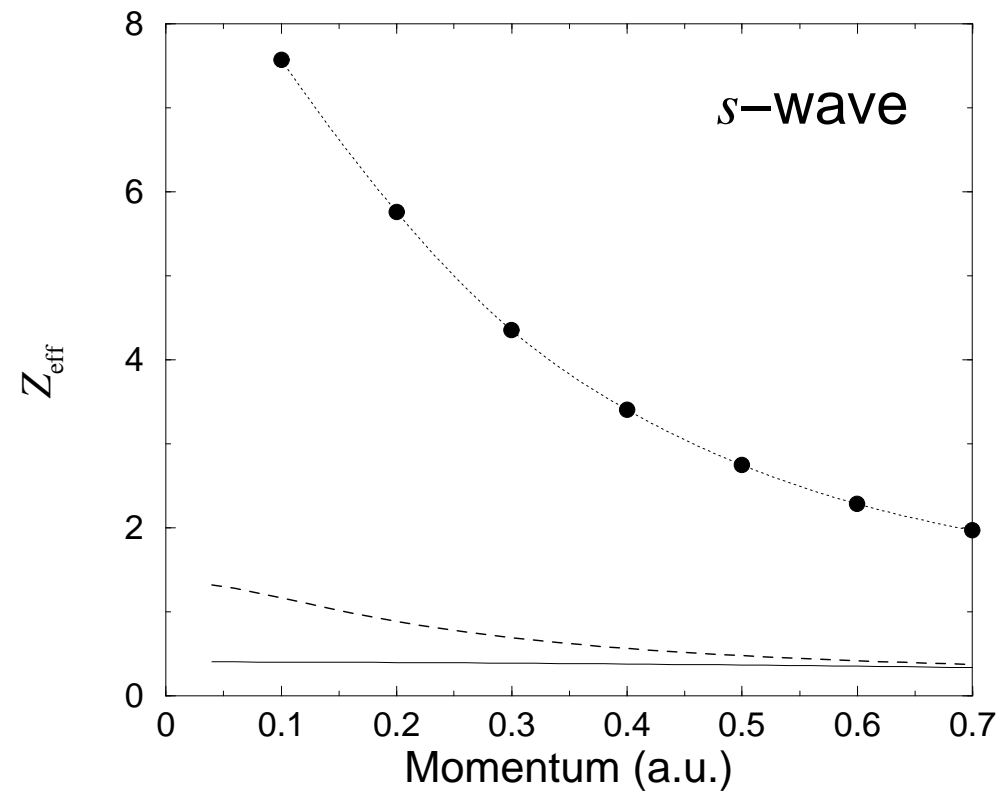

FIG. 10: Contribution of the 0th order diagram, Fig. 5(a), to the positron-hydrogen $s$-wave annihilation parameter $Z_{\text {eff }}$, calculated with the positron wavefunction in the static atomic potential (solid curve), and with the Dyson orbital (dashed curve). Circles connected by dotted curve are the accurate results of Refs. [57].

The difficulty of calculating the vertex corrections to $Z_{\text {eff }}$ accurately is evident from these graphs, as all the diagrams in Fig. [5] contribute significantly. The higher-order diagrams containing the vertex function, are close to or greater than the lower-order diagrams. Note that all contributions have a similar dependence on the positron momentum. It is driven by the momentum dependence of the positron Dyson orbitals (external lines in the diagrams), as discussed in Sec. ID

Figure[1] shows that for $p$ and $d$ waves, the contribution of the diagram Fig. [5 (f) grows rapidly and becomes largest towards the Ps formation threshold. This diagram describes annihilation inside the virtual Ps formation, which has a vigorous energy dependence close to threshold [48]. This may have a bearing on the kinks in $Z_{\text {eff }}$ visible at $k \approx 0.6$ a.u. for the $p$ and $d$ waves. Although they may be a numerical artifact, an indication of an inflection point is also present in the accurate $p$-wave results of Ref. [57].

The final results for $R=15$ a.u. are slightly higher than for $R=30$ a.u. Indeed, annihilation takes place near the atom, and a denser knot sequence for $R=15$ may provide a better description of small electron-positron separations. Our final values compare well with the accurate positron-hydrogen results obtained using a variational approach [57]. In that work the electron-positron distance was represented explicitly in the calculation, while we use a single-centre expansion. We believe that the remaining small discrepancy could be eliminated by 'pushing harder' the numerics in our approach. Thus, Fig. 12 shows that if we use $n=60 \mathrm{~B}$-splines of order $k=9$ and include the first 23 basis states, the difference between our $Z_{\text {eff }}$ and the benchmark values is halved.

The main conclusion of this section is that the numerical implementation of the many-body theory approach proposed in this paper works. For positron collisions with hydrogen, where this approach is exact, the calculations reproduce the best scattering phaseshifts, and yield good results in the more difficult annihilation problem.

\section{CORRELATION CONTRIBUTION TO THE ANNIHILATION VERTEX.}

The ability of a many-body theory to describe correlation corrections to the annihilation vertex may give some insight into the role such corrections play in positron annihilation with matter. Thus, in theoretical studies of positron annihilation in condensed matter, one usually starts by performing a calculation for the ground state electron and positron densities. The annihilation rate is then found via an equation of the form (see, e.g., $[\underline{6}]$ ),

$$
\lambda=\pi r_{0}^{2} c \int \rho_{e}(\mathbf{r}) \rho_{p}(\mathbf{r}) \gamma\left(\rho_{e}, \rho_{p}\right) \mathrm{d} \mathbf{r},
$$



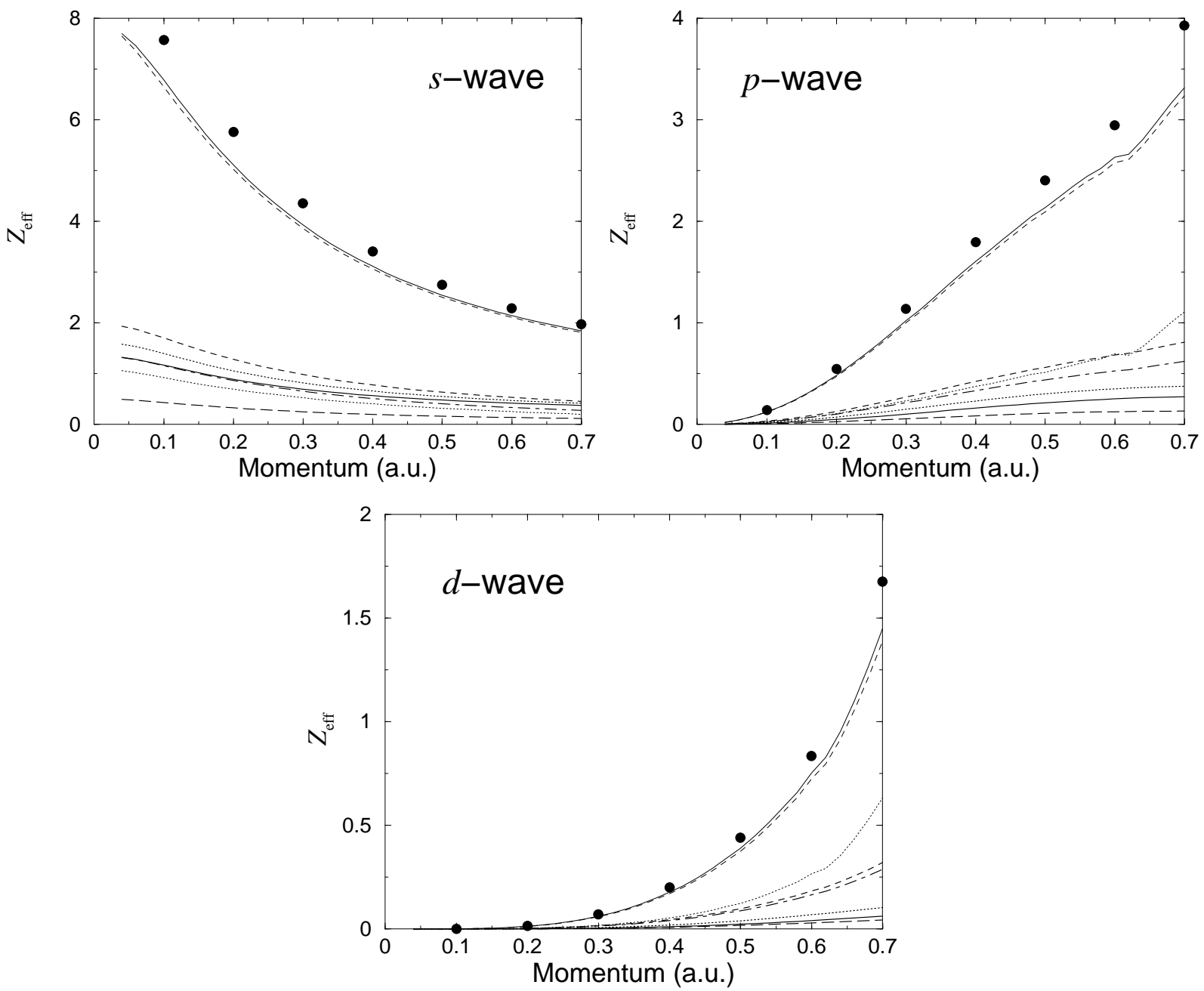

FIG. 11: Annihilation parameter $Z_{\text {eff }}$ for the $s$-, $p$-, and $d$-wave positron on hydrogen. Contributions of individual diagrams from Fig. 5 are: thick solid line, diagram (a); thick dotted line, (b); long dashed line, (c); thick dashed line, (d); dot-dashed line, (e); thin dotted line, (f) (all for $R=15$ a.u.). Thin solid line is the total for $R=15$ a.u., thin dashed line, total for $R=30$ a.u. Circles are the results of Ref. 57].

where $\rho_{p}(\mathbf{r})$ and $\rho_{e}(\mathbf{r})$ are the positron and electron densities and $\gamma\left(\rho_{e}, \rho_{p}\right)$ is the so-called enhancement factor. It is introduced to account for the Coulomb attraction in the annihilating electron-positron pair. It has long been known that the independent-particle approximation $(\gamma=1)$ underestimates the annihilation rates by several times [59], and a number of semi-empirical and interpolation forms of $\gamma\left(\rho_{e}, \rho_{p}\right)$ have been suggested (see, e.g., Ref. [60] and references therein).

A comparison between Eq. (37) with $\gamma=1$ and the many-body theory expression (24) for the annihilation rate, shows that the former corresponds to the 0th-order term in $Z_{\text {eff }}$, Eq. [22) [1]. As we have seen in Sec. [IV] (Fig. [10), $Z_{\text {eff }}^{(0)}$ does underestimate the annihilation rate in hydrogen by a factor of 5 , even when the best positron wavefunction is used. On the other hand, the correlation correction to the annihilation rate [2nd term in Eq. (24)] does not have the form of Eq. (37). This correction depends on the positron wavefunction at two different points, $\mathbf{r}$ and $\mathbf{r}^{\prime}$. Therefore, any local expression like Eq. (37) is necessarily an approximation.

To illustrate this point, a contour plot in Fig. 13 shows the radial part of the integrand of the nonlocal term,

$$
P_{\varepsilon l}(r) \Delta_{\varepsilon}^{(l)}\left(r, r^{\prime}\right) P_{\varepsilon l}\left(r^{\prime}\right),
$$

for the $s$-wave $(l=0)$ positron annihilation on hydrogen at $k=0.06$ a.u. The plot confirms that the correlation contribution to the annihilation vertex is localised near the atom. Its maximum at $r=r^{\prime} \approx 1.5$ a.u. compares well with the radius of the hydrogen atom, and the ridge-like structure indicates that the "nonlocality" is limited to $\left|r-r^{\prime}\right| \sim 1$ a.u. 


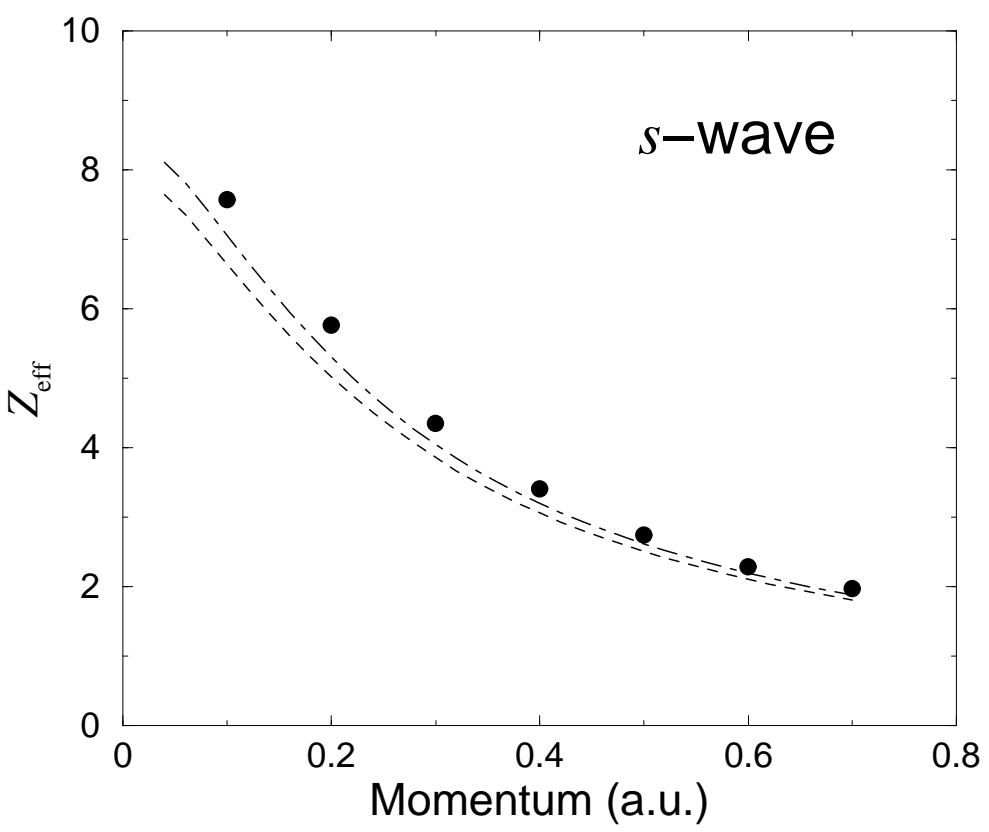

FIG. 12: Positron annihilation on hydrogen, $s$ wave. Many-body theory results: dashed line, $R=30$ a.u., $n=40, k=6$, first 15 states used; dot-dashed line, $R=30$ a.u., $n=60, k=9$, first 23 states used; circles, Ref. 57].

The overall size of the (nonlocal) correction to the annihilation vertex can be characterised by the average enhancement factor $\bar{\gamma}$,

$$
\bar{\gamma}=1+\frac{\int \psi_{\varepsilon}^{*}(\mathbf{r}) \Delta_{\varepsilon}\left(\mathbf{r}, \mathbf{r}^{\prime}\right) \psi_{\varepsilon}\left(\mathbf{r}^{\prime}\right) d \mathbf{r} d \mathbf{r}^{\prime}}{\int \sum_{n}\left|\varphi_{n}(\mathbf{r})\right|^{2}\left|\psi_{\varepsilon}(\mathbf{r})\right|^{2} d \mathbf{r}}
$$

We defined this factor in such a way that when used in place of $\gamma$ in Eq. (37), together with $\rho_{e}(\mathbf{r})=\sum_{n}\left|\varphi_{n}(\mathbf{r})\right|^{2}$ and $\rho_{p}(\mathbf{r})=\left|\psi_{\varepsilon}(\mathbf{r})\right|^{2}$, it would reproduce correct values of the positron-atom annihilation rate.

The quantity $\bar{\gamma}$ can also be defined as the ratio of the total $Z_{\text {eff }}$ to the value obtained from the 0th-order diagram, $Z_{\text {eff }}^{(0)}$, Fig. 囵(a). Because of the weak energy dependence of $\Delta_{\varepsilon}$ (see Sec. [ID), this ratio should not depend strongly on the energy of the incident positron. This also implies that $\bar{\gamma}$ will be relatively insensitive to the wavefunction used to describe the incident positron. In particular, the use of either HF or Dyson wavefunctions $\psi_{\varepsilon}$ for the incident positron should yield close values of $\bar{\gamma}$.

As a test, this ratio was evaluated for the annihilation of the $s, p$ and $d$-wave positrons on hydrogen, see Fig. 14 As expected, the values of $\bar{\gamma}$ depend weakly on the incident positron energy, except when the Ps formation threshold is approached. This confirms the earlier observation that the momentum dependence of various contributions to $Z_{\text {eff }}$ in Fig. 11 is approximately the same. The results obtained within the static approximation and with the Dyson orbitals are also very close to each other, even though the absolute values of $Z_{\text {eff }}$ obtained in the two approximations are very different (see, e.g., Fig. 10). One cannot help noticing that the enhancement due to annihilation vertex corrections increases with the angular momentum of the positron. For larger $l$, the penetration of the positron into the electron-rich regions of the atom is suppressed by the centrifugal barrier, and the effect of "pulling the electron out" (i.e., virtual Ps formation), described by the correlation corrections, becomes more important.

Equation (37) is local, whereas equation (24) contains a non-local term. In order to compare the enhancement of $Z_{\text {eff }}$ due to this term with the enhancement factors used in condensed matter calculations, we need to "localise" the contribution of $\Delta_{\varepsilon}\left(\mathbf{r}, \mathbf{r}^{\prime}\right)$. Let us do this by introducing an effective 'electron correlation density' $\tilde{\rho}_{e}(r)$ via the relation

$$
\iint P_{\varepsilon l}(r) \Delta_{\varepsilon}^{(l)}\left(r, r^{\prime}\right) P_{\varepsilon l}\left(r^{\prime}\right) d r d r^{\prime} \equiv \int \tilde{\rho}_{e}(r) P_{\varepsilon l}^{2}(r) d r,
$$

for positron annihilation in the $l$ th partial wave. This allows us to define an effective enhancement factor $\gamma_{e}(r)$ through

$$
\rho_{e}(r)+\tilde{\rho}_{e}(r) \equiv \gamma_{e}(r) \rho_{e}(r),
$$




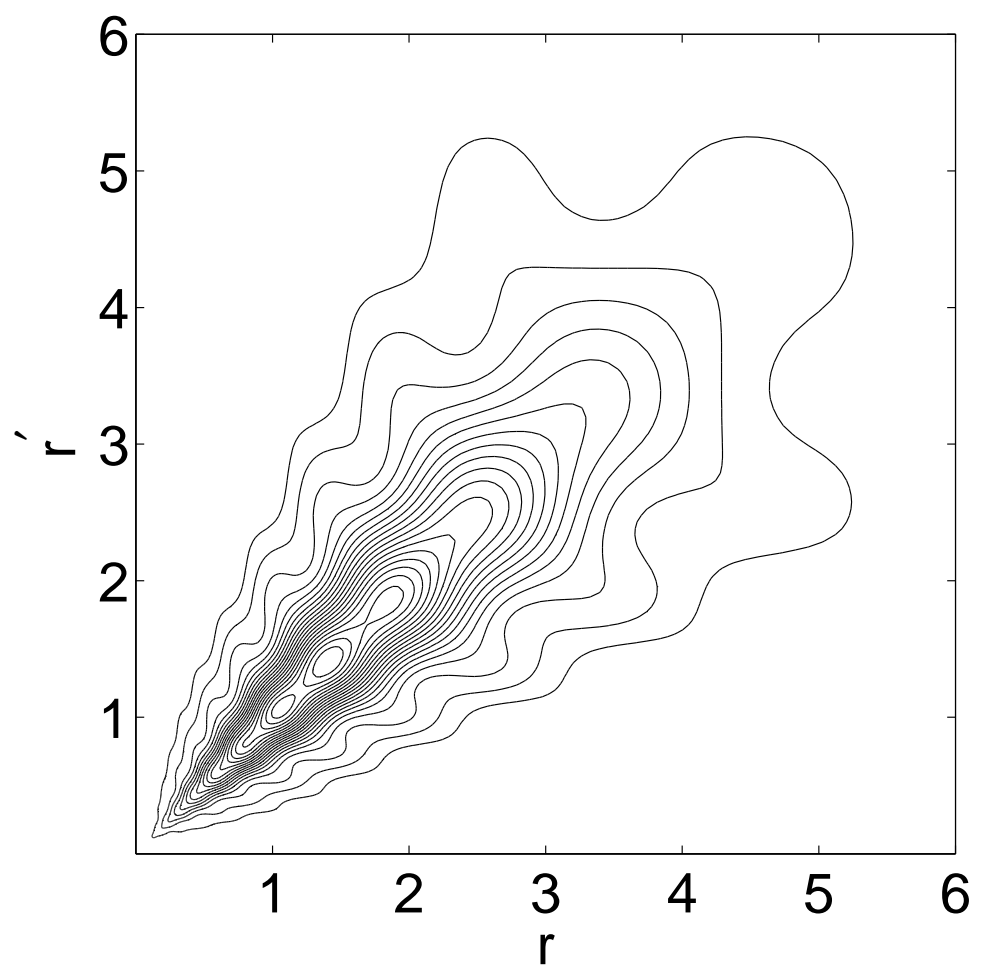

FIG. 13: Radial dependence of the integrand (38) of the correlation correction for the annihilation of the $s$-wave positron with momentum $k=0.06$ a.u. on hydrogen. The "ripples" is an artifact of the reconstruction of $\Delta_{\varepsilon}^{(l)}\left(r, r^{\prime}\right)$ from the matrix element $\left\langle i\left|\Delta_{\varepsilon}\right| j\right\rangle$.

which is equivalent to

$$
\gamma_{e}(r)=1+\tilde{\rho}_{e}(r) / \rho_{e}(r)
$$

where $\rho_{e}(r)=P_{1 s}^{2}(r) / 4 \pi r^{2}$, and $P_{1 s}=2 r e^{-r}$ for hydrogen. Equations (40) and (42) guarantee that if we use $\gamma_{e}(r)$ in Eq. (37), correct annihilation rates will be recovered.

Equation (40) does not define $\tilde{\rho}_{e}(r)$ uniquely. We use two different methods to obtain it numerically. The first one states,

$$
\tilde{\rho}_{e}^{[1]}(r)=\frac{\int \Delta_{\varepsilon}^{(l)}\left(r, r^{\prime}\right) P_{\varepsilon l}\left(r^{\prime}\right) d r^{\prime}}{P_{\varepsilon l}(r)}
$$

which ensures that $\tilde{\rho}_{e}^{[1]}(r)$ satisfies Eq. (40) exactly. However, it has a disadvantage in that it may have unphysical poles at the zeroes of the positron wavefunction. A second method involves calculating

$$
\tilde{\rho}_{e}^{[2]}(r)=\int_{-2 r}^{2 r} \Delta_{\varepsilon}^{(l)}(r+\sigma / 2, r-\sigma / 2) d \sigma
$$

which follows from Eq. (40) if we change variables $r, r^{\prime}$ to $r \pm \sigma / 2$, and keep the lowest order term in the Taylor expansion of $P_{\varepsilon l}(r+\sigma / 2) P_{\varepsilon l}(r-\sigma / 2)$. This approximation may not be accurate for small $r$, where the positron wavefunction varies rapidly, but should be correct for larger $r$ values, where the peaking of $\Delta_{\varepsilon}^{(l)}\left(r, r^{\prime}\right)$ at $r=r^{\prime}$ (Fig. 13) means that $P_{\varepsilon l}(r)$ varies slowly on the scale of typical $\sigma$.

Figure [15] shows both electron correlation densities calculated for the $s$-wave positron with momentum $k=0.5$ a.u. Apart from the small range of distances near the origin, the values of $\tilde{\rho}_{e}$ from the two methods are close, although $\tilde{\rho}_{e}^{[2]}$ shows some numerical "noise" related to the reconstruction of $\Delta_{\varepsilon}^{(l)}\left(r, r^{\prime}\right)$ from its matrix elements. A comparison with the hydrogen ground-state electron density shows that the latter drops much faster with the distance from the nucleus. In fact, $\tilde{\rho}_{e}$ is much greater than $\rho_{e}$ at those $r$ where the positron density is large, in agreement with the correlation contribution to $Z_{\text {eff }}$ being five times $Z_{\text {eff }}^{(0)}$. 


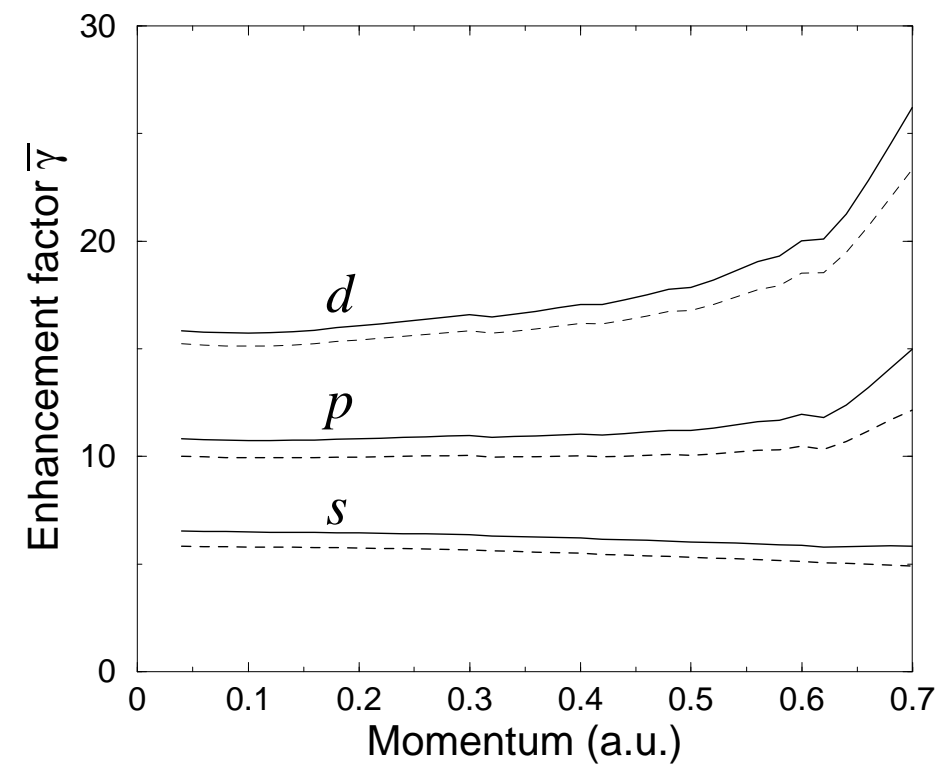

FIG. 14: Ratio $\bar{\gamma}$ of the total $Z_{\text {eff }}$ to $Z_{\text {eff }}^{(0)}$ for positron annihilation on hydrogen. Values obtained with positron wavefunction in the static approximation are shown by solid curves, and those with the Dyson orbitals, by dashed curves.

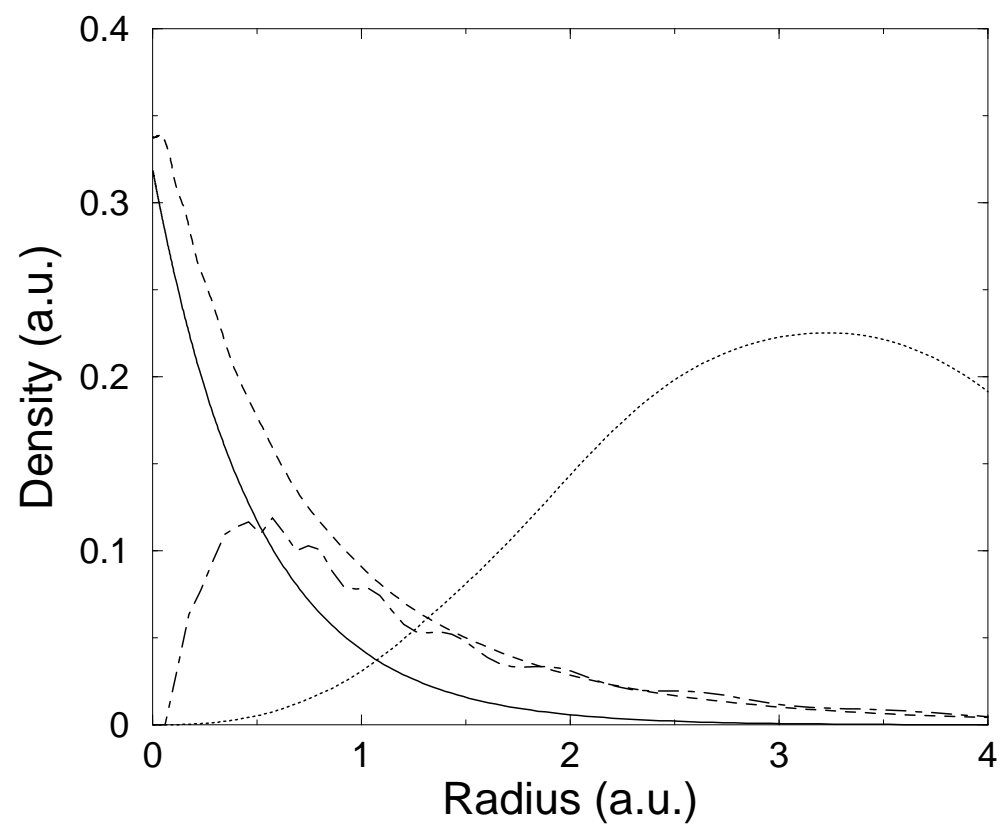

FIG. 15: Effective electron correlation densities $\tilde{\rho}_{e}^{[1]}(r)$ (dashed curve) and $\tilde{\rho}_{e}^{[2]}(r)$ (dot-dash curve) for the annihilation of the $s$-wave positron with $k=0.5$ on hydrogen. Shown for comparison are the ground-state electron density in hydrogen $\rho_{e}(r)$ (solid curve), and the positron density $P_{\varepsilon l}^{2}(r)$ (dotted curve, arbitrarily scaled).

The electron correlation densities obtained above allow us to calculate the corresponding enhancement factors and compare them with a parametrisation of $\gamma\left(\rho_{e}, \rho_{p}\right)$ derived by Arponen and Pajanne 62] for a positron in a homogeneous electron gas,

$$
\gamma_{\mathrm{AP}}=1+1.23 r_{s}-0.0742 r_{s}^{2}+\frac{1}{6} r_{s}^{3},
$$


where $r_{s}$ is a measure of the average distance between the electrons,

$$
r_{s}=\left(\frac{3}{4 \pi \rho_{e}}\right)^{1 / 3}
$$

This enhancement factor is plotted in Fig. [16 together with the effective enhancement factors $\gamma_{e}(r)$ derived from Eq. (42) using $\tilde{\rho}_{e}^{[1]}$, which is more stable numerically than $\tilde{\rho}_{e}^{[2]}$. Note that unlike $\gamma_{\mathrm{AP}}$, the factor $\gamma_{e}$ obtained from our many-body theory approach, depends on the energy and orbital angular momentum of the positron. The values shown in Fig. [16 correspond to the momenta, $k=0.06$ ( $s$ wave) and 0.5 a.u. ( $s, p$ and $d$ waves).

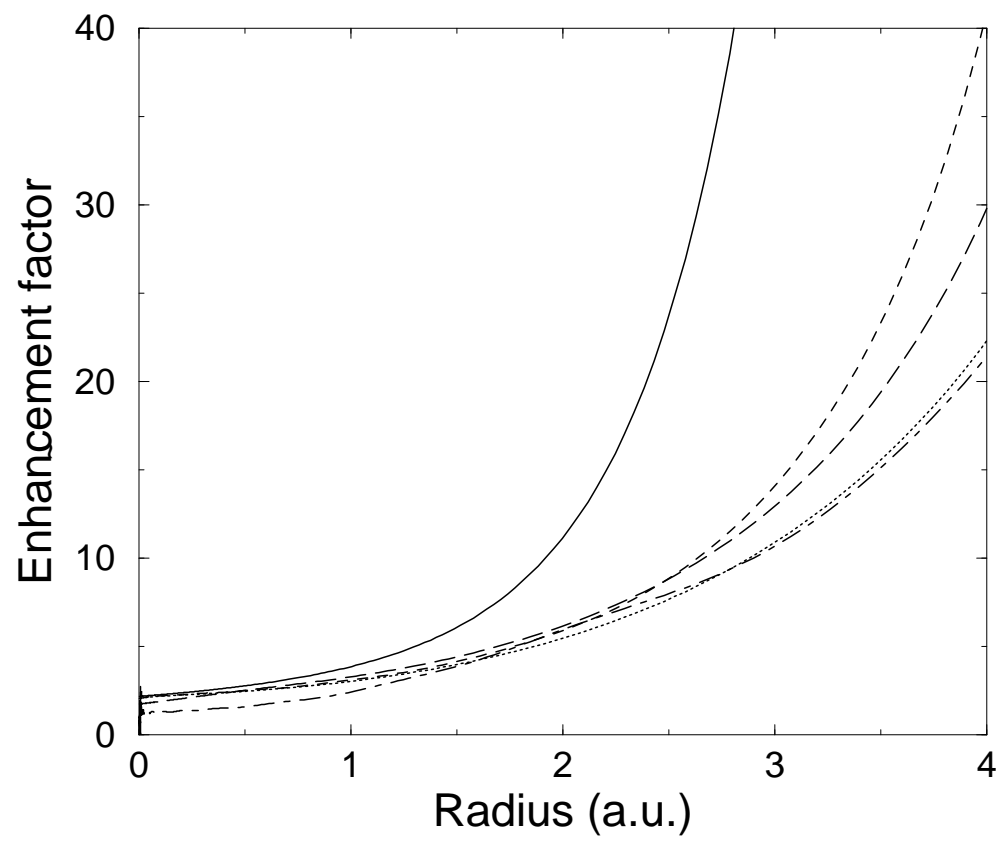

FIG. 16: Enhancement factors for positron annihilation on hydrogen. Solid curve, $\gamma_{A P}$, Eq. 45); dotted line $\gamma_{e}$ for $s$-wave positrons at $k=0.06$; dashed line, $\gamma_{e}$ for $s$-wave positrons at $k=0.5$; long-dashed line, $\gamma_{e}$ for $p$-wave positrons at $k=0.5$; dot-dashed line, $\gamma_{e}$ for $d$-wave positrons at $k=0.5$.

A feature common to all enhancement factors in Fig. [16 is their rapid rise with the distance from the nucleus. This increase is related to the drop of the electron density, a relation which is explicit in Eq. 455 for $\gamma_{\mathrm{AP}}$. At small distances, where the electron density is large, $\gamma_{\mathrm{AP}}$ compares well with $\gamma_{e}$. However, at larger distances, where the electron density is low, $\gamma_{\mathrm{AP}}$ is much greater than all of the $\gamma_{e}$. Such a discrepancy could be expected, given that a homogeneous electron gas theory used to derive $\gamma_{\mathrm{AP}}$ is more reliable in the high-density limit. The exaggeration of the enhancement by $\gamma_{\mathrm{AP}}$ was reported in Ref. [60] where various forms of the enhancement factor were tested by comparison with accurate annihilation rates for a number of positron-atom bound systems. For Be and $\mathrm{Mg}$ (which have $I>6.8 \mathrm{eV}$ ), the values obtained using $\gamma_{\mathrm{AP}}$ overestimated the accurate annihilation rates by factors of 5 and 2 , respectively. It must be noted that the product $\rho_{e} \gamma_{\mathrm{AP}}$ remains finite as $\rho_{e} \rightarrow 0$. This means that one cannot in principle use it in Eq. (37) for a continuous spectrum positron, since it would yield infinite values of the annihilation rate and $Z_{\text {eff. }}$. For the same reason a much stronger overestimate observed in Ref. [60] with $\gamma_{\mathrm{AP}}$ for Be is a direct consequence of the positron binding energy for Be being much smaller than for $\mathrm{Mg}$.

Figure 16 confirms that $\gamma_{e}$ is energy-dependent. It is greater for $k=0.5$ a.u. compared with $k=0.06$ ( $s$ wave), the higher momentum being closer to the Ps formation threshold. Values of $\gamma_{e}$ derived for the $p$ and $d$ waves are similar to those from the $s$ wave. Hence, the large differences between the average enhancement factors in Fig. 14 are due to the effect of the centrifugal barrier on the positron wavefunction. The distances which effectively contribute to $Z_{\text {eff }}$ are greater for the positron in higher partial waves. 


\section{SUMMARY AND OUTLOOK}

In this paper we have formulated a many-body theory approach which accounts for the main correlation effects in positron-atom interactions. These are (i) polarisation of the target by the positron, (ii) virtual positronium formation, and (iii) strong enhancement of the electron-positron contact density due to their Coulomb interaction. The key development for an accurate description of (ii) and (iii) is the summation of the ladder diagram series and calculation of the electron-positron vertex function. B-spline basis sets and extrapolation over the orbital angular momenta are used to achieve convergence of the sums over the electron and positron intermediate states. The method can be applied to a range of problems such as positron scattering, annihilation and formation of bound states.

Although our main interest is in exploring many-electron targets, the method has been first tested for hydrogen, where accurate benchmark data exist for the scattering phaseshifts and annihilation rates. In the case of hydrogen the present formalism is exact. Numerically, excellent agreement with accurate variational calculations for the phase shifts has been obtained, together with a good agreement for the annihilation parameter $Z_{\text {eff }}$. The calculation of the most difficult part of the correlation potential, which contains the vertex function, for many-electron atoms is only marginally more difficult than for hydrogen. Therefore, we expect that application of our many-body theory to the problems of positron scattering and annihilation on noble-gas atoms and binding to halogen ions 63, 64] should yield accurate results. In particular, we would like to re-examine and improve the accuracy of the many-body theory predictions [33] of positron binding energies to the $n s^{2}$ atoms such as $\mathrm{Mg}, \mathrm{Cd}$ and $\mathrm{Zn}$.

The advantage of many-body theory methods is their physical transparency. It allows one to distinguish between different physical mechanisms and compare their relative importance. Thus, we saw that virtual Ps formation in positron-hydrogen scattering is just as important as the target polarisation. Correlation corrections to the annihilation vertex, which are physically related to the virtual Ps formation, are even more important. They enhance the annihilation rate in hydrogen by a factor of 5 or more, depending on the positron partial wave. Such vertex corrections depend weakly on the positron energy, and the enhancement they produce is practically the same for various positron wavefunctions. Therefore, one could use the average enhancement factors derived for isolated atoms to obtain reliable annihilation rates for atoms placed in different environments, provided that a sufficiently accurate single-particle positron wavefunction is available. Of course, different atomic subshells will be characterised by different enhancement factors. However, they can all be determined in an many-body calculations of the type described in this paper, and serve as an input for the calculations of positron annihilation in molecules or condensed matter.

In this work we have analysed the spatial dependence of the nonlocal correlation corrections to the annihilation vertex. We have also derived the equivalent local enhancement factor and compared it with an expression used in condensed matter calculations. Similar comparisons for larger many-electron targets may test various forms of enhancement factors in a much greater range of electron densities.

The rapid development of computers over the past few decades seems to have favoured theoretical methods other than many-body theory. Such methods often rely more on the computer power and numerical techniques than on the physical insight. They often appear to be "more exact" than the sophisticated but explicitly approximate many-body theory approaches, and promise improved results due to shear growth of computer power. Their drawback is that they do not always increase one's physical understanding of the problem. We believe that a further theoretical development of many-body methods combined with a judicial use of computers is a healthy alternative.

\section{Acknowledgments}

GG is grateful to V. V. Flambaum who drew the positron-atom problem to his attention while at the University of New South Wales (Sydney), and to W. R. Johnson for pointing out the advantages of B-spline bases. The work of JL has been supported by the award from the Department of Employment and Learning (Northern Ireland). We also thank P. Van Reeth for providing data for positron annihilation on hydrogen in numerical form.

\section{APPENDIX A: TECHNICAL DETAILS}

For a positron interacting with a spherically-symmetric target, both the self energy $\Sigma_{E}\left(\mathbf{r}, \mathbf{r}^{\prime}\right)$ and the correlation correction to the annihilation vertex $\Delta_{E}\left(\mathbf{r}, \mathbf{r}^{\prime}\right)$ can be expanded in partial waves, e.g.,

$$
\Sigma_{E}\left(\mathbf{r}, \mathbf{r}^{\prime}\right)=\frac{1}{r r^{\prime}} \sum_{\lambda=0}^{\infty} \sum_{\mu=-\lambda}^{\mu=\lambda} Y_{\lambda \mu}(\Omega) \Sigma_{E}^{(\lambda)}\left(r, r^{\prime}\right) Y_{\lambda \mu}^{*}\left(\Omega^{\prime}\right) .
$$


Defining the volume element $d \mathbf{r}=r^{2} d r d \Omega$, and the positron wavefunction with orbital angular momentum $l, \varphi_{\varepsilon}(\mathbf{r})=$ $r^{-1} P_{\varepsilon l}(r) Y_{l m}(\Omega)$, we obtain the matrix element $\left\langle\varepsilon|\Sigma| \varepsilon^{\prime}\right\rangle$ as,

$$
\begin{aligned}
\left\langle\varepsilon\left|\Sigma_{E}\right| \varepsilon^{\prime}\right\rangle & =\int \varphi_{\varepsilon}^{*}(\mathbf{r}) \Sigma_{E}\left(\mathbf{r}, \mathbf{r}^{\prime}\right) \varphi_{\varepsilon^{\prime}}\left(\mathbf{r}^{\prime}\right) d \mathbf{r} \\
& =\int P_{\varepsilon l}(r) \Sigma_{E}^{(l)}\left(r, r^{\prime}\right) P_{\varepsilon^{\prime} l}\left(r^{\prime}\right) d r d r^{\prime} .
\end{aligned}
$$

The angular reduction of the various diagrams in $\left\langle\varepsilon\left|\Sigma_{E}\right| \varepsilon^{\prime}\right\rangle$ and $\left\langle\varepsilon\left|\Delta_{E}\right| \varepsilon\right\rangle$ is simplified by the use of graphical techniques for performing angular momentum algebra [65]. The final expressions for the diagrams in terms of reduced matrix elements, $3 j$ and $6 j$ symbols are given below.

The reduced Coulomb matrix element is defined as,

$$
\begin{aligned}
\left\langle 3,4\left\|V_{l}\right\| 2,1\right\rangle & =\sqrt{\left[l_{1}\right]\left[l_{2}\right]\left[l_{3}\right]\left[l_{4}\right]}\left(\begin{array}{ccc}
l_{1} & l & l_{3} \\
0 & 0 & 0
\end{array}\right)\left(\begin{array}{ccc}
l_{2} & l & l_{4} \\
0 & 0 & 0
\end{array}\right) \\
& \times \int P_{\varepsilon_{3} l_{3}}\left(r_{1}\right) P_{\varepsilon_{4} l_{4}}\left(r_{2}\right) \frac{r_{<}^{l}}{r_{>}^{l+1}} P_{\varepsilon_{2} l_{2}}\left(r_{2}\right) P_{\varepsilon_{1} l_{1}}\left(r_{1}\right) d r_{1} d r_{2},
\end{aligned}
$$

where $\left[l_{1}\right] \equiv 2 l_{1}+1$, etc. Note that instead of including the minus sign in the reduced Coulomb matrix element when it involves the positron, we account for it in the overall sign factor for the diagram (see below). The reduced Coulomb matrix element for an electron-positron pair coupled into a total angular momentum $J$ is given by

$$
\left\langle 3,4\left\|V^{(J)}\right\| 2,1\right\rangle=\sum_{l}(-1)^{J+l}\left\langle 3,4\left\|V_{l}\right\| 2,1\right\rangle\left\{\begin{array}{lll}
J & l_{3} & l_{4} \\
l & l_{2} & l_{1}
\end{array}\right\} .
$$

This expression is similar to the 'exchange' matrix element that one meets in all-electron problems [22].

The sum of the ladder diagram series (the vertex function) is calculated via the matrix equation (6), which must be solved for all possible total angular momenta $J$ of the electron-positron pair:

$$
\left\langle\nu_{2}, \mu_{2}\left\|\Gamma_{E}^{(J)}\right\| \mu_{1}, \nu_{1}\right\rangle=-\left\langle\nu_{2}, \mu_{2}\left\|V^{(J)}\right\| \mu_{1}, \nu_{1}\right\rangle-\sum_{\nu, \mu} \frac{\left\langle\nu_{2}, \mu_{2}\left\|V^{(J)}\right\| \mu, \nu\right\rangle\left\langle\nu, \mu\left\|\Gamma_{E}^{(J)}\right\| \mu_{1}, \nu_{1}\right\rangle}{E-\varepsilon_{\nu}-\varepsilon_{\mu}} .
$$

In the Appendix, the state labels $\nu, \nu_{1}$, etc., refer to the positron orbitals $\varepsilon_{\nu} l_{\nu}, \varepsilon_{\nu_{1}} l_{\nu_{1}}$, etc. Similarly, $\mu$, $\mu_{1}$, etc., label excited-state electron orbitals. Electron orbitals occupied in the target ground state (holes) are labeled by Latin indices $(n)$. When B-spline basis states are used, Eq. (A5) is a finite-dimension matrix equation solved by matrix inversion.

The self-energy diagrams can be expressed in terms of these matrix elements. For closed-shell atoms, each loop in the diagram gives a spin factor of 2 . This factor should be omitted for hydrogen which has only one electron in the $1 s$ orbital. The sign factor for each diagram is $(-1)^{a+b+c}$, where $a$ is the number of hole lines, $b$ is the number of electron-hole loops and $c$ is the number of positron-electron Coulomb interactions. In the expressions below we label the incident positron angular momentum by $l_{p}$. This is also the orbital angular momentum of the external lines $\varepsilon, \varepsilon^{\prime}$ of the diagrams.

The 2nd-order self-energy diagram, Fig. 1(a), is given by

$$
2 \sum_{\nu, \mu, n} \sum_{l} \frac{\left\langle\varepsilon^{\prime}, n\left\|V_{l}\right\| \mu, \nu\right\rangle\left\langle\nu, \mu\left\|V_{l}\right\| n, \varepsilon\right\rangle}{[l]\left[l_{p}\right]\left(E+\varepsilon_{n}-\varepsilon_{\nu}-\varepsilon_{\mu}\right)} .
$$

The virtual-Ps contribution to $\Sigma_{E}$, Fig. [2] is obtained after finding the vertex function as follows:

$$
2 \sum_{\nu_{i}, \mu_{i}, n} \sum_{J} \frac{[J]\left\langle\varepsilon^{\prime}, n\left\|V^{(J)}\right\| \mu_{2}, \nu_{2}\right\rangle\left\langle\nu_{2}, \mu_{2}\left\|\Gamma_{E+\varepsilon_{n}}^{(J)}\right\| \mu_{1}, \nu_{1}\right\rangle\left\langle\nu_{1}, \mu_{1}\left\|V^{(J)}\right\| n, \varepsilon\right\rangle}{\left[l_{p}\right]\left(E+\varepsilon_{n}-\varepsilon_{\nu_{1}}-\varepsilon_{\mu_{1}}\right)\left(E+\varepsilon_{n}-\varepsilon_{\nu_{2}}-\varepsilon_{\mu_{2}}\right)} .
$$

Matrix elements of the annihilation $\delta$-function are defined similarly to the Coulomb ones, using the expansion of the $\delta\left(\mathbf{r}_{1}-\mathbf{r}_{2}\right)$ in terms of spherical harmonics [65]. The reduced matrix element then is

$$
\begin{aligned}
\left\langle 3,4\left\|\delta_{l}\right\| 2,1\right\rangle & =\frac{[l]}{4 \pi} \sqrt{\left[l_{1}\right]\left[l_{2}\right]\left[l_{3}\right]\left[l_{4}\right]}\left(\begin{array}{ccc}
l_{1} & l & l_{3} \\
0 & 0 & 0
\end{array}\right)\left(\begin{array}{ccc}
l_{2} & l & l_{4} \\
0 & 0 & 0
\end{array}\right) \\
& \times \int P_{\varepsilon_{3} l_{3}}(r) P_{\varepsilon_{4} l_{4}}(r) P_{\varepsilon_{2} l_{2}}(r) P_{\varepsilon_{1} l_{1}}(r) r^{-2} d r
\end{aligned}
$$


and the matrix element for an electron-positron pair coupled into a total angular momentum $J$ is given by,

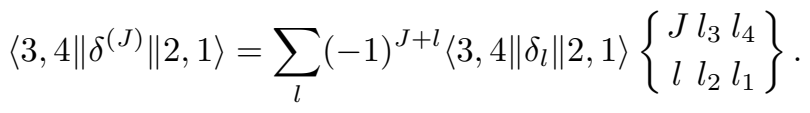

The various diagrams contributing to $Z_{\text {eff }}$ can then be expressed in terms of these matrix elements. As with the self energy diagrams, the factor of 2 which results from closed loops, must be removed for hydrogen. Note that in the diagrams below, we also include factors of 2 to account for the mirror images of those diagrams that are not symmetric. To produce correct values of $Z_{\text {eff }}$ the expressions given below must be multiplied by the positron normalisation factor (25).

The 0th-order diagram, Fig. $4(\mathrm{a})$, is a sum of simple radial integrals over all hole orbitals $n$,

$$
2 \sum_{n} \frac{\left[l_{n}\right]}{4 \pi} \int P_{\varepsilon l_{p}}^{2}(r) P_{\varepsilon_{n} l_{n}}^{2}(r) r^{-2} d r
$$

The 1st-order contribution, Fig. 4(b) and (c), is given by

$$
-4 \sum_{\nu, \mu, n} \sum_{l} \frac{\left\langle\varepsilon, n\left\|\delta_{l}\right\| \mu, \nu\right\rangle\left\langle\nu, \mu\left\|V_{l}\right\| n, \varepsilon\right\rangle}{[l]\left[l_{p}\right]\left(E+\varepsilon_{n}-\varepsilon_{\nu}-\varepsilon_{\mu}\right)}
$$

Expressions for the remaining four contributions, Fig. 5](c)-(f), are:

$$
\begin{gathered}
2 \sum_{\nu_{i}, \mu_{i}, n} \sum_{J} \frac{[J]\left\langle\varepsilon, n\left\|V^{(J)}\right\| \mu_{2}, \nu_{2}\right\rangle\left\langle\nu_{2}, \mu_{2}\left\|\delta^{(J)}\right\| \mu_{1}, \nu_{1}\right\rangle\left\langle\nu_{1}, \mu_{1}\left\|V^{(J)}\right\| n, \varepsilon\right\rangle}{\left[l_{p}\right]\left(E+\varepsilon_{n}-\varepsilon_{\nu_{2}}-\varepsilon_{\mu_{2}}\right)\left(E+\varepsilon_{n}-\varepsilon_{\nu_{1}}-\varepsilon_{\mu_{1}}\right)}, \\
\quad-4 \sum_{\nu_{i}, \mu_{i}, n} \sum_{J} \frac{[J]\left\langle\varepsilon, n\left\|\delta^{(J)}\right\| \mu_{2}, \nu_{2}\right\rangle\left\langle\nu_{2}, \mu_{2}\left\|A_{E+\varepsilon_{n}}^{(J)}\right\| n, \varepsilon\right\rangle}{\left[l_{p}\right]\left(E+\varepsilon_{n}-\varepsilon_{\nu_{2}}-\varepsilon_{\mu_{2}}\right)}, \\
4 \sum_{\nu_{i}, \mu_{i}, n} \sum_{J} \frac{[J]\left\langle\varepsilon, n\left\|V^{(J)}\right\| \mu_{3}, \nu_{3}\right\rangle\left\langle\nu_{3}, \mu_{3}\left\|\delta^{(J)}\right\| \mu_{2}, \nu_{2}\right\rangle\left\langle\nu_{2}, \mu_{2}\left\|A_{E+\varepsilon_{n}}^{(J)}\right\| n, \varepsilon\right\rangle}{\left[l_{p}\right]\left(E+\varepsilon_{n}-\varepsilon_{\nu_{3}}-\varepsilon_{\mu_{3}}\right)\left(E+\varepsilon_{n}-\varepsilon_{\nu_{2}}-\varepsilon_{\mu_{2}}\right)}, \\
2 \sum_{\nu_{i}, \mu_{i}, n} \sum_{J} \frac{[J]\left\langle\varepsilon, n\left\|A_{E+\varepsilon_{n}}^{(J)}\right\| \mu_{3}, \nu_{3}\right\rangle\left\langle\nu_{3}, \mu_{3}\left\|\delta^{(J)}\right\| \mu_{2}, \nu_{2}\right\rangle\left\langle\nu_{2}, \mu_{2}\left\|A_{E+\varepsilon_{n}}^{(J)}\right\| n, \varepsilon\right\rangle}{\left[l_{p}\right]\left(E+\varepsilon_{n}-\varepsilon_{\nu_{3}}-\varepsilon_{\mu_{3}}\right)\left(E+\varepsilon_{n}-\varepsilon_{\nu_{2}}-\varepsilon_{\mu_{2}}\right)},
\end{gathered}
$$

where we have introduced

$$
\left\langle\nu_{2}, \mu_{2}\left\|A_{E+\varepsilon_{n}}^{(J)}\right\| n, \varepsilon\right\rangle=\sum_{\nu_{1}, \mu_{1}} \frac{\left\langle\nu_{2}, \mu_{2}\left\|\Gamma_{E+\varepsilon_{n}}^{(J)}\right\| \mu_{1}, \nu_{1}\right\rangle\left\langle\nu_{1}, \mu_{1}\left\|V^{(J)}\right\| n, \varepsilon\right\rangle}{E+\varepsilon_{n}-\varepsilon_{\nu_{1}}-\varepsilon_{\mu_{1}}}
$$

This amplitude not only helps to make the expressions above more compact. It also provides a practical way of computing these diagrams. Thus, without a preliminary evaluation of $\left\langle\nu_{2}, \mu_{2}\left\|A_{E+\varepsilon_{n}}^{(J)}\right\| n, \varepsilon\right\rangle$, diagram (f) in Fig. 5 contains eight-fold summation over hundreds of electron and positron basis states.

[1] W. J. Cody, J. Lawson, H. S. W. Massey, and K. Smith, Proc. Roy. Soc. A 278, 479 (1964).

[2] M. Ya. Amusia, N. A. Cherepkov, L. V. Chernysheva and S. G. Shapiro, J. Phys. B 9, L531 (1976).

[3] V. A. Dzuba, V. V. Flambaum, W. A. King, B. N. Miller, and O. P. Sushkov, Phys. Scr. T46, 248 (1993).

[4] G. F. Gribakin and W. A. King, J. Phys. B 27, 2639 (1994).

[5] New Directions in Antimatter Chemistry and Physics, edited by C. M. Surko and F. A. Gianturco (Kluwer Academic Publishers, Dordrecht, 2001).

[6] M. J. Puska and R. M. Nieminen, Rev. Mod. Phys. 66, 841 (1994).

[7] A. David, G. Kögel, P. Sperr and W. Triftshäuser, Phys. Rev. Lett. 87, 067402 (2001). 
[8] G. Jerusalem, R. Hustinx, Y. Beguin and G. Fillet, Eur. J. Cancer 39, 1525 (2003).

[9] C. Schwartz, Phys. Rev. 124, 1468 (1961).

[10] A. K. Bhatia, A. Temkin, R. J. Drachman, and H. Eiserike, Phys. Rev. A 3, 1328 (1971); A. K. Bhatia, A. Temkin, and H. Eiserike Phys. Rev. A 9, 219 (1974).

[11] J. W. Humberston, J. Phys. B, 6, L305 (1973); R. I. Campeanu and J. W. Humberston, ibid. 10, L153 (1977).

[12] J. W. Humberston, Adv. At. Mol. Phys. 15, 101 (1979).

[13] J. W. Humberston, P. Van Reeth, M. S. T. Watts and W. E. Meyerhof, J. Phys. B 30, 2477 (1997).

[14] P. Van Reeth and J. W. Humberston, J. Phys. B 32, 3651 (1999).

[15] M. T. McAlinden, A. A. Kernoghan, and H. R. J. Walters, Hyperfine Interactions 89, 161 (1994); J. Phys. B 29, 555 (1996); 29, 3971 (1996); 30, 1543 (1997).

[16] G. G. Ryzhikh, J. Mitroy, and K. Varga, J. Phys. B 31, 3965 (1998).

[17] V. A. Dzuba, V. V. Flambaum, G. F. Gribakin and C. Harabati, Phys. Rev. A 60, 3641 (1999); V. A. Dzuba, V. V. Flambaum and C. Harabati, Phys. Rev. A 62, 042504 (2000).

[18] I. A. Ivanov, J. Mitroy, and K. Varga, Phys. Rev. Lett. 87, 063201 (2001); J. Mitroy and I. A. Ivanov, Phys. Rev. A 65, 012509 (2002).

[19] M. W. J. Bromley and J. Mitroy, Phys. Rev. A 65, 062505 (2002); 65, 062506 (2002).

[20] M. W. J. Bromley and J. Mitroy, Phys. Rev. A 66, 062504 (2002); 67, 062709 (2003).

[21] A. L. Fetter and J. D. Walecka, Quantum Theory of Many-Particle Systems (McGraw-Hill, New York, 1971).

[22] M. Ya. Amusia and N. A. Cherepkov, Case Studies in Atomic Physics 5, 47 (1975).

[23] H. P. Kelly, Phys. Rev. 131, 684 (1963).

[24] M. Ya. Amusia, N. A. Cherepkov, L. V. Chernysheva, S. G. Shapiro, and A. Tanchich, Zh. Eksp. Teor. Fiz. 68, 2023 (1975) [Sov. Phys. JETP 411012 (1975)].

[25] M. Ya. Amusia, N. A. Cherepkov, L. V. Chernysheva, D. M. Davidović and V. Radojević, Phys. Rev. A 25, 219 (1982). According to this paper the coefficient in Eq. (15) is $-\pi$, because they express the self-energy operator $\Sigma_{E}$ in Rydberg.

[26] W. R. Johnson and C. Guet, Phys. Rev. A 49, 1041 (1994); 64, 019901 (2001).

[27] L. V. Chernysheva, G. F. Gribakin, V. K. Ivanov and M. Yu. Kuchiev, J. Phys. B 21, L419 (1988); G. F. Gribakin, B. V. Gultsev, V. K. Ivanov and M. Yu. Kuchiev, J. Phys. B 23, 4505 (1990).

[28] W. R. Johnson, J. Sapirstein, and S. A. Blundell, J. Phys. B 22, 2341 (1989).

[29] V. A. Dzuba, V. V. Flambaum, G. F. Gribakin and O. P. Sushkov, Phys. Rev. A 44, 2823 (1991).

[30] V. A. Dzuba and G. F. Gribakin, Phys. Rev. A 49, 2483 (1994); 50, 3551 (1994).

[31] V. A. Dzuba, V. V. Flambaum, P. G. Silvestrov and O. P. Sushkov, J. Phys. B 20, 1399 (1987); Phys. Lett. A 131, 461 (1988); V. A. Dzuba, V. V. Flambaum and O. P. Sushkov, Phys. Lett. A 140, 493 (1989).

[32] S. A. Blundell, W. R. Johnson and J. Sapirstein, Phys. Rev. A 38, 4961 (1988).

[33] V. A. Dzuba, V. V. Flambaum, G. F. Gribakin and W. A. King, Phys. Rev. A 52, 4541 (1995).

[34] V. A. Dzuba, V. V. Flambaum, G. F. Gribakin and W. A. King, J. Phys. B 29, 3151 (1996).

[35] G. F. Gribakin and W. A. King, Can. J. Phys. 74, 449 (1996).

[36] G. G. Ryzhikh and J. Mitroy, Phys. Rev. Lett. 79, 4124 (1997).

[37] G. G. Ryzhikh and J. Mitroy, J. Phys. B 31, L401 (1998); J. Mitroy and G. G. Ryzhikh, ibid. 32, 1375 (1999).

[38] J. Mitroy, M. W. J. Bromley and G. G. Ryzhikh, J. Phys. B 35, R81 (2002).

[39] J. Mitroy, M. W. J. Bromley and G. G. Ryzhikh, in Ref. [5], pp. 199-221.

[40] A. B. Migdal, Theory of Finite Fermi-Systems and Applications to Atomic Nuclei (Interscience, New York, 1967).

[41] J. S. Bell and E. J. Squires, Phys. Rev. Lett. 3, 96 (1959).

[42] L. D. Landau and E. M. Lifshitz, Quantum Mechanics, 3rd ed. (Pergamon, Oxford, 1977).

[43] For electrons this also means the absence of the contributions of the target exchange potential.

[44] RPA provides reliable values of atomic dipole polarisabilities, see, e.g., M. Ya. Amus'ya, N. A. Cherepkov, and S. G. Shapiro, Zh. Eksp. Teor. Fiz. 63, 889 (1972) [Sov. Phys. JETP 36, 468 (1973)]; D. Kolb, W. R. Johnson, and P. Shorer, Phys. Rev. A 26, 19 (1982); W. R. Johnson, D. Kolb, and K.-N. Huang, At. Data and Nucl. Data Tables 28, 333 (1983).

[45] G. F. Gribakin, in Photonic, Electronic and Atomic Collisions (XXII ICPEAC), Proceedings, Eds. J. Burgdorfer, J. Cohen, S. Datz, and C. Vane, (Rinton Press, Princeton, 2002), pp. 353-64.

[46] P. A. Fraser, Adv. At. Mol. Phys. 4, 63 (1968).

[47] This cross section is given by $\bar{\sigma}_{2 \gamma}=\pi r_{0}^{2}(c / v)$, where $v$ is the electron-positron relative velocity, see, e.g., V. B. Berestetskii, E. M. Lifshitz, and L. P. Pitaevskii, Quantum electrodynamics (Pergamon, Oxford, 1982). Annihilation into two gamma quanta is allowed only for the zero total spin of the pair, $S=0$. For $S=1$ they annihilate into three photons. The corresponding spin-averaged cross section is almost 400 times smaller: $\bar{\sigma}_{3 \gamma}=\left[4\left(\pi^{2}-9\right) / 3\right] \alpha r_{0}^{2}(c / v)$, where $\alpha=e^{2} / \hbar c \approx$ $1 / 137$.

[48] G. F. Gribakin and J. Ludlow, Phys. Rev. Lett. 88, 163202 (2002); J. Ludlow and G. F. Gribakin, Phys. Rev. A 66, 064704 (2002).

[49] J. Sapirstein and W. R. Johnson, J. Phys. B 29, 5213 (1996).

[50] C. de Boor, A Practical Guide to Splines (Springer, New York, 1978).

[51] M. Ya. Amusia, L. V. Chernysheva, Computation of Atomic Processes. A Handbook for the ATOM Programs (IOP Publishing, Bristol, 1997).

[52] I. Bray and A. T. Stelbovics, Comp. Phys. Comm. 85, 1 (1995); I. Bray and D. V. Fursa, Phys. Rev. Lett. 76, 2674 (1996).

[53] I. Bray and A. T. Stelbovics, Phys. Rev. A 48, 4787 (1993).

[54] J Mitroy and G Ryzhikh, J. Phys. B 32, 2831 (1999). 
[55] G. F. Gribakin and J. Ludlow, J. Phys. B 35, 339 (2002).

[56] C. Schwartz, Phys. Rev. 126, 1015 (1962); Methods in Computational Physics, Vol. 2 (Academic, New York, 1962), pp. 241-266.

[57] P. Van Reeth and J. W. Humberston, J. Phys. B 31, L231 (1998).

[58] G. F. Gribakin, Phys. Rev. A 61, 022720 (2000).

[59] R. A. Ferrell, Rev. Mod. Phys. 28, 308 (1956).

[60] J. Mitroy and B. Barbiellini, Phys. Rev. B 65, 235103 (2002).

[61] This is true to the extent that we neglect the small difference between the true and HF electron densities, and that between $\left|\psi_{\varepsilon}(\mathbf{r})\right|^{2}$ and $\rho_{p}(\mathbf{r})$.

[62] J. Arponen and E. Pajanne, Ann. Phys. 121343 (1979). See also B. Barbiellini, M. J. Puska, T. Torsti, and R. M. Nieminen, Phys. Rev. B 51, 7341 (1986).

[63] J. Ludlow and G. F. Gribakin, to be submitted to Phys. Rev. A (2004).

[64] J. Ludlow and G. F. Gribakin, to be submitted to J. Phys. B (2004).

[65] D. A. Varshalovich, A. N. Moskalev and V. K. Khersonskii, Quantum Theory of Angular Momentum (World Scientific, Singapore, 1988). 